\title{
Ultrasound Evaluation of Complications of Extracranial Carotid Artery Interventions
}

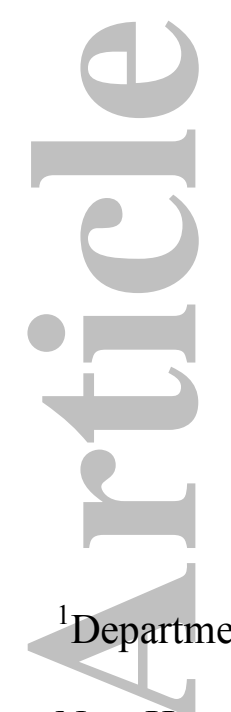

New Haven, Connecticut, USA

\author{
John D. Millet, MD, MHS ${ }^{1,2}$ \\ Joseph J. Cavallo, MD ${ }^{1}$ \\ Leslie M. Scoutt, MD ${ }^{1}$ \\ Gowthaman Gunabushanam, $\mathrm{MD}^{1}$
}

Please address correspondence to:

Gowthaman Gunabushanam, MD

Assistant Professor, Department of Radiology and Biomedical Imaging

Yale University School of Medicine

333 Cedar St, P O Box 208042

New Haven, CT. 06520

Tel: +1-203-506-1275

Fax: +1-203-785-3061

Email: gowthaman7@gmail.com

This is the author manuscript accepted for publication and has undergone full peer review but has not been through the copyediting, typesetting, pagination and proofreading process, which may lead to differences between this version and the Version record. Please cite this article as doi:10.1002/ jum.14376. 
LMS is an educational consultant for Philips Healthcare (not related to this manuscript).

Short running title: Complications of Carotid Artery Interventions

Manuscript category: Pictorial Essay

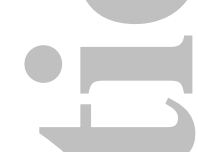

\section{Summary}

Carotid endarterectomy and carotid artery stenting are among the most common peripheral vascular procedures performed worldwide. Ultrasound is the initial, and often only imaging modality used in the evaluation of iatrogenic carotid arterial injuries. This pictorial review provides an overview of the clinical, and sonographic findings of complications following interventions in the extracranial carotid arteries, including dissection, fluid collections, pseudoaneurysm, thrombosis, thromboembolism, restenosis, and stent deformation. Grey scale, color, and pulsed Doppler imaging findings are reviewed and correlation with $\mathrm{CT}, \mathrm{MRI}$, and angiography are provided.

Key words: carotid artery, complications, stenting, endarterectomy, sonography

\section{Introduction}

Carotid endarterectomy (CEA) and carotid artery stenting (CAS) are among the most common peripheral vascular procedures performed worldwide. ${ }^{1,2}$ In the United States, it is estimated that 1,350,000 CEAs were performed and 90,000 CASs were placed between 1998-2008. ${ }^{3}$ During this same time period, CAS utilization in the United 
States increased from $2.8 \%$ to $12.6 \%$ of all carotid artery revascularization procedures, ${ }^{3}$ due in part to the publication of several large, multi-center studies demonstrating its safe use as a less-invasive alternative to CEA for certain patient populations. ${ }^{4,5}$

Ultrasound is considered the first-line imaging modality of choice for the evaluation of iatrogenic vascular injuries. As surgical and endovascular carotid artery interventions have become increasingly common, sonologists may expect to encounter iatrogenic complications from these procedures. Additionally, intraoperative ultrasound is performed following CEA at some institutions to assess for the presence of residual intimal flaps, residual stenosis and to exclude carotid arterial wall thrombus. ${ }^{6,7}$ The purpose of this article is to provide a pictorial review of complications following surgical and percutaneous endovascular interventions in the extracranial carotid arteries (Table 1). The sonographic appearances of these complications are reviewed and illustrative CT, MRI and/or angiographic correlation provided.

\section{Dissection}

Arterial dissections result from tears between any of the three arterial wall layers (intima, media and adventitia). Depending on whether or not there is an exit as well as entrance tear, this may result in an intramural hematoma or creation of a false lumen from blood flowing into the defect. Dissections may also result from spontaneous rupture of a vasa vasorum, with the direct introduction of blood between vessel layers. ${ }^{8}$ The incidence of spontaneous carotid dissection has been estimated at 2.6-2.9 per 100,000 patients in all age groups. ${ }^{9,10}$ In a retrospective analysis of 6,981 diagnostic and therapeutic interventions in the carotid and vertebral arteries, iatrogenic arterial dissections occurred 
in $0.25 \%$ of cases with 10 of 18 reported dissections occurring in the internal carotid arteries. ${ }^{11}$ In this series, no dissections were observed in the pediatric population and the incidence increased to $0.35 \%$ in patients over 35 years of age. ${ }^{11}$ Iatrogenic causes of carotid dissections include vessel layer disruption during CEA and other open procedures of the neck or skull base, ${ }^{12,13}$ intimal trauma from guidewire manipulation during percutaneous endovascular procedures, ${ }^{11}$ trauma to the vessel wall from the contrast injection jet, ${ }^{11}$ and inadvertent direct puncture of the carotid artery during attempt at central venous catheterization. ${ }^{12}$ While carotid dissection is more common with increasing age, ${ }^{11}$ other associated vascular risk factors include hypertension, diabetes, smoking, hyperlipidemia and use of oral contraceptives. ${ }^{14}$ Genetic connective tissue diseases that cause arteriopathy or weakness of the vessel wall, including Ehlers-Danlos syndrome, Marfan's syndrome, and autosomal dominant polycystic kidney disease, also increase dissection risk. ${ }^{13}$ A history of recent infection, especially respiratory infection has been shown to be more prevalent in patients with carotid artery dissection. ${ }^{15}$ Although intraprocedural dissections are often immediately identified, carotid dissections can cause a range of symptoms including localized and unilateral neck, facial, or orbital pain, headaches (most commonly frontotemporal), partial Horner's Syndrome (unilateral miosis and ptosis) or cranial nerve palsies (most commonly hypoglossal). ${ }^{16}$ Stroke is the most feared consequence of carotid artery dissection. Dissection-related strokes are most commonly embolic in nature, but can also be ischemic due to decreased blood flow and thrombus formation within the true lumen, which can result in complete thrombosis or occlusion of the carotid artery. ${ }^{17}$ One meta-analysis that included 180 patients with acute strokes in the setting of carotid artery dissection demonstrated similar 
safety and outcomes with the use of thrombolytics as in other stroke patients. ${ }^{18}$

Antithrombotic therapy for 3-6 months with aspirin or a combination of heparin and warfarin has been advocated to reduce the risk of thromboembolism, and both treatments have shown similar efficacy in preventing recurrent stroke and disability. ${ }^{19,20}$ Patients with evidence of expanding dissections, symptoms despite anticoagulation, or contraindications to anticoagulation may benefit from endovascular or surgical repair. ${ }^{8}$ (

On grey scale imaging, a carotid artery dissection is characterized by the presence of a mobile, echogenic flap within the artery or false lumen. When thrombosed, the false lumen can appear hypoechoic or echogenic and may or may not encroach on the true lumen. A thrombosed false lumen can be difficult to differentiate from an intramural hematoma or plaque, both of which may also narrow the true lumen. Color Doppler can demonstrate flow within a patent false lumen parallel to the true lumen, separated by a thin echogenic linear structure representing the intima. The spectral Doppler waveforms observed in carotid arterial dissections are variable and may be normal, damped (lower amplitude with a biphasic pattern), high resistance, or absent flow. ${ }^{14}$ Although magnetic resonance angiography (MRA) and computed tomographic angiography (CTA) may also be used to diagnose and evaluate carotid arterial dissections, ${ }^{21}$ ultrasound is better suited for serial imaging to follow resolution or progression.

When intraoperative ultrasound is done, small mobile flaps may be seen within the artery on grey scale ultrasound (Figure 1). Typically, an arteriotomy followed by exploration is done if the flap is mobile and $2 \mathrm{~mm}$ or greater in length in the internal carotid artery, $3 \mathrm{~mm}$ or greater in the common carotid artery, or if there is a technical 
defect with greater than $30 \%$ luminal diameter reduction. ${ }^{6,7}$ Intimal flaps and residual disease are usually left undisturbed in the external carotid artery.

\section{Fluid collections}

Fluid collections, including hematomas, seromas, and abscesses, are well-known complications of CEA. Cervical hematomas can vary in size and severity and are dangerous due to their close vicinity to structures vital to respiration and cerebral blood flow and their ability to quickly expand.

In a randomized clinical trial, wound hematomas were found to occur in $7.1 \%$ (101 of 1415) patients that underwent CEA. ${ }^{22}$ While the majority of wound complications were minor, $3.3 \%$ of patients (46 of 1415 ) required surgical reexploration, including four patients $(0.3 \%)$ who died as a direct consequence of the wound hematomas. ${ }^{22}$ Cervical hematomas following CEA are associated with increased risk of mortality, operative stroke, operative myocardial infarction, and blood transfusion, and an increased length of hospital stay. ${ }^{23}$ While a rare occurrence, the wires utilized during CAS can perforate branches of the external carotid artery leading to hematoma formation. ${ }^{24}$ In a systematic review and meta-analysis of randomized clinical trials comparing CAS to CEA, the 30day risk for hematoma formation was lower with CAS with a reported odds ratio of $0.59 .^{25}$ Anticoagulation is a well-known risk factor for hematoma formation during carotid artery procedures. In an analysis of 4,587 CEAs, reversal of heparin anticoagulation with protamine during CEA significantly reduced the incidence of postCEA bleeding requiring reoperation from 1.66 to $0.64 \%$ without increasing the risk of myocardial infarction, stroke, or death. ${ }^{26}$ Other risk factors for hematoma formation after 
CEA include intraoperative hypotension and carotid shunt placement. ${ }^{17}$ Acute and large hematomas are potentially hazardous entities and when not addressed in a timely manner can lead to stroke (Figure 2). Some hematomas may resolve without intervention;

however many surgeons will elect for immediate surgical revision when a hematoma is diagnosed. $^{27}$

Abscesses can result from superinfection of a post-CEA hematoma or may occur as a result of an infected carotid patch. The incidence of carotid patch infections is rare at $0.25-0.5 \%,{ }^{28}$ and may be greater when prosthetic patches are used. ${ }^{29}$ Neck swelling and formation of a local draining sinus or a pseudoaneurysm are the most common presentations of an infected carotid patch, with half of patients presenting 3 months postoperatively. ${ }^{30}$

On grey scale ultrasound, hematomas are usually visualized as a soft tissue mass with heterogeneous echogenicity, often adjacent to nearby vessels (Figure 3).

Echogenicity varies depending on the age of the hematoma. Fluid-fluid levels may be observed (Figure 2). Absence of flow should be confirmed on color and pulsed Doppler. Abscesses can present as a poorly defined heterogeneously echogenic mass. In some cases, punctate echogenic foci representing gas bubbles may be seen (Figure 4).

\section{Pseudoaneurysm}

A pseudoaneurysm (PSA) typically results from disruption of all three layers of the arterial wall resulting in a jet or extravasation of blood through the neck of the PSA into the adjacent sac, which is contained by a compacted layer of the surrounding soft tissues. Partial thrombosis of the PSA sac is common. However, some PSAs arise 
secondary to weakening of the arterial wall from damage to only one or two layers, for example following a dissection, resulting in an outpouching or focal bulge of the remainder of the arterial wall, usually the adventitial layer and sometimes the media into the surrounding tissues. ${ }^{31}$ Iatrogenic PSAs of the carotid arteries are rare. The incidence of PSA following CEA has been reported to be $0.37 \%$, with underlying causes including suture failure, arterial wall degeneration, injury to the vessel wall from surgical clamping, dissection, and infection. ${ }^{32}$ Additional cases have been reported following carotid stenting ${ }^{33}$ and placement of central lines. ${ }^{34}$ Risk factors for iatrogenic PSA formation include advanced age, female gender, calcified vessels, obesity, and anticoagulation. ${ }^{31}$

A carotid PSA may present immediately following an inciting event or years later. ${ }^{35}$ In one reported case, a man presented with a PSA 29 years after CEA. ${ }^{36}$ Presenting symptoms may include pulsatile neck swelling, palpable cervical mass, pain, persistent bleeding, or infection at the surgical site. ${ }^{32}$ PSAs may also present with neurological symptoms due to compression of nearby nerves and can be found in asymptomatic patients undergoing follow up imaging. ${ }^{35}$ Traditionally, treatment for carotid PSAs has involved open surgery. ${ }^{35}$ However, endovascular repair is becoming more common. A recent systematic review of endovascular stenting for true and false aneurysm repair found it comparable in efficacy to open repair. ${ }^{37}$

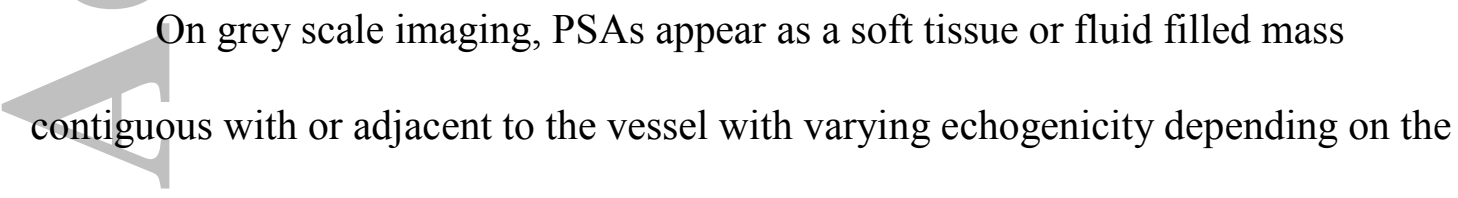
presence or lack of thrombus. Persistent swirling blood flow on color Doppler within the PSA sac, with a "yin-yang” pattern, can help distinguish a PSA from a hematoma. The neck connecting the PSA to the carotid artery may be wide or thin, depending on etiology. 
If thin, a typical "to-and-fro" waveform pattern characterized by flow heading towards the PSA during systole and away from the PSA during diastole will be observed. Angiography, CT, or MRI can be useful to confirm the diagnosis of a carotid PSA, size, presence or absence of intraluminal thrombus and anatomy (width and length) of the neck, all of which are important for determining optimal patient management (Figure 5).

\section{Thrombosis and thromboembolism}

Carotid thrombosis and thromboembolism leading to stroke are among the most feared complications of carotid artery interventions and may occur independently or in conjunction with one of the other complications described above. The long-term (greater than 2 years) incidence of major ipsilateral stroke following revascularization ranges from $0.5-3.0 \%$ for CEA and $1.2-4.6 \%$ for CAS, with no significant difference yet observed between the two approaches. ${ }^{38}$ However, studies have found the long-term risk of all strokes (major and minor) to be significantly higher for CAS than CEA, ${ }^{39,40}$ with incidence reported to range from $7.9-10.1 \%$ for CEA and $9.0-14.2 \%$ for $\mathrm{CAS}^{38} \mathrm{~A}$ few trials have also reported a significantly higher rate of stroke in the periprocedure period for CAS, ${ }^{39-41}$ although this risk may be mitigated by the use of embolic protective devices during stenting. ${ }^{38}$

Acute thrombosis is a catastrophic event that requires immediate recognition and treatment with thrombolysis, endovascular thrombosuction, or open surgery. ${ }^{42}$ The most frequently cited causes of acute thrombosis following CAS are failure to receive antiplatelet medication and technical failure, such as stent malposition and carotid dissection. ${ }^{43}$ Risk factors for stroke following CEA include female sex,${ }^{44}$ non-Caucasian 
race, prior stroke or transient ischemic attack, contralateral stenosis $>50 \%$, severe disability, and coronary artery disease, ${ }^{45}$ while risk factors for stroke following CAS include advanced age ( $\geq 80$ years), chronic renal failure, diabetes mellitus, symptomatic status, operator inexperience, and use of multiple stents. ${ }^{46}$ High grade stenosis and heavily calcified plaque are also believed to increase the risk of stroke.

On ultrasound, acute carotid thrombus appears as a hypoechoic area within the vessel or stent lumen. A "string sign," demonstrating a thin string-like line of residual blood flow on color Doppler imaging, may be seen adjacent to the area of thrombus (Figure 6). High resistance waveforms may be observed in the common carotid artery proximal to the site of thrombus. Flow velocities may be elevated on spectral Doppler ultrasound at the site of thrombus. When the thrombus completely occludes the vessel or stent lumen, no blood flow will be detected. Downstream cerebral ischemia resulting from thromboembolism may be seen with other imaging modalities, such as diffusionweighted brain MRI (Figure 7).

\section{Restenosis}

The incidence of restenosis following CEA and CAS has been reported to range from $5 \%$ to $20 \%$, with variation in rates between studies influenced in part by the duration of follow-up and the exact definition of restenosis that is used. ${ }^{47}$ Although some studies have reported significantly higher rates of restenosis with CAS than with CEA, ${ }^{25,48}$ the Carotid Revascularization Endarterectomy versus Stenting Trial found no significant difference in rates between the two procedures, with restenosis rates after 2 
years reported at $6.0 \%$ and $6.3 \%$ for CAS and CEA, respectively. ${ }^{47}$ Patients who develop restenosis after CEA are also likely to develop in-stent restenosis following CAS. ${ }^{49}$

Risk factors for the development of restenosis following CEA or CAS include female sex, diabetes, dyslipidemia. ${ }^{47,50-52}$ Coronary artery disease and smoking have been identified as additional risk factors for restenosis after CEA, ${ }^{47,53}$ while advanced age, the use of balloon-expandable stents, and implantation of multiple stents may contribute to in-stent restenosis following CAS. ${ }^{54}$ Other risk factors include fibrous or inflammatory plaque, history of prior neck radiation therapy, prior history of restenosis following CEA, residual waisting or narrowing immediately post treatment, and increased age. There is currently no clear consensus on how to manage restenosis following carotid revascularization and follow-up imaging should therefore be handled on an individualized basis. ${ }^{55-57}$ Generally, a baseline US is obtained, followed by US every 6 months for the first 2 years, and yearly thereafter. ${ }^{47}$ If US shows moderate stenosis, continued follow-up every 6 months is done.

On grey scale ultrasound, stenosis appears as a focal area of luminal narrowing within the carotid vessel or stent lumen, possibly with wall thickening and plaque. Blood flow velocities will be elevated at the point of stenosis on spectral Doppler, and color Doppler may demonstrate narrowing of the lumen and color aliasing (Figure 8). Peak systolic velocity at the site of the stenosis is useful in determining the degree of stenosis ${ }^{58}$ There are no widely accepted Doppler criteria for grading restenosis following CEA and CAS. To compound matters further, no clear consensus exists as to what percentage or degree of recurrent stenosis following CEA or CAS should be treated. 
Following CAS, when evaluating for in-stent restenosis, the rigid stent-arterial wall complex can cause higher overall peak systolic velocities. ${ }^{51,59}$ Armstrong et al ${ }^{60}$ used stent peak systolic velocity (PSV) greater than $300 \mathrm{~cm} / \mathrm{s}$, end diastolic velocity (EDV) more than $125 \mathrm{~cm} / \mathrm{s}$ and ratio of PSV in-stent divided by proximal common carotid artery $>4$ to diagnose $>75 \%$ stenosis. Criteria used for more than $50 \%$ stent stenosis was a PSV $>150 \mathrm{~cm} / \mathrm{s}$ and a PSV stent ratio $>2$. Other published criteria include stent PSV $>300 \mathrm{~cm} / \mathrm{s}$ and PSV $>450 \mathrm{~cm} / \mathrm{s}$ for diagnosing $70 \%$ stenosis within the stent. $^{61,62}$ It is often helpful to obtain baseline measurements to later assess the change in PSV over time. In case of doubt, one must have a lower threshold for obtaining a confirmatory CTA, MRA, or angiogram. Following CEA, Doppler velocity criteria are likely to be lower as placing an endarterectomy patch in most cases increases the diameter of the carotid bulb, thereby lowering PSV and vessel wall compliance likely increases.

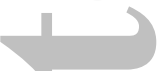

\section{Stent deformation and fracture}

Carotid stents are exposed to a wide range of forces that over time can result in stent deformation or fracture. ${ }^{63}$ Deformation is defined as stent shape and caliber distortions without disruption of stent struts, while fracture results from strut failure and breakage. $^{21,64}$ In a case series that followed 116 carotid stents yearly for up to 9 years, the prevalence of deformation $(23 \%)$ was greater than that of fracture $(4 \%)$ and the incidence of both increased over time. ${ }^{64}$ Risk of stent deformation and fracture may increase with local vessel calcification, ${ }^{65}$ vessel tortuosity, ${ }^{66}$ and a history of local radiation therapy. ${ }^{63}$ The use of open-cell design stents may help to mitigate fracture risk in patients with 
heavily calcified or tortuous vessels due to their increased flexibility. ${ }^{66}$ Although stent fractures are usually treated conservatively, yearly imaging follow-up has been recommended because of the increased risk of restenosis. ${ }^{63}$ Restenosis rates have been reported to range from $12.5 \%-21 \%$ in patients with confirmed carotid stent fracture. ${ }^{63,65}$ On grey scale ultrasound, properly deployed, well-functioning stents appear as thin, parallel echogenic lines adjacent to the intima of the vessel wall. Malapposition between the stent and the vessel wall with intervening flow on Doppler imaging is indicative of mechanical stent failure. Bends appear as wavy irregularities throughout the stent wall (Figure 9), while fractures may be seen as stent wall disruptions with or without protrusion of fragmented struts into the vessel lumen. These findings can be confirmed with plain radiographs, digital subtraction angiography, or CT. In some cases, the intimal flap of a dissection may be confused with stent fracture, especially in the setting of a highly calcified dissecting flap.

r

\section{Arteriovenous fistula}

Arteriovenous fistula has been described as an extremely rare complication following CEA. ${ }^{67}$ In a case report, an arteriovenous fistula was noted to have developed between the external carotid artery to the internal jugular vein approximately 6 years following CEA. ${ }^{67}$ This was treated by using coil embolization of the fistula and adjoining portion of the internal jugular vein.

\section{Conclusion}


Ultrasound is often the initial imaging modality used in the evaluation of many iatrogenic carotid arterial injuries, whether clinically suspected or found incidentally at routine follow up imaging. Knowledge of the sonographic appearance of complications following carotid arterial interventions is essential to their early detection, may enable a reduction in patient morbidity and mortality, and help determine the optimal management
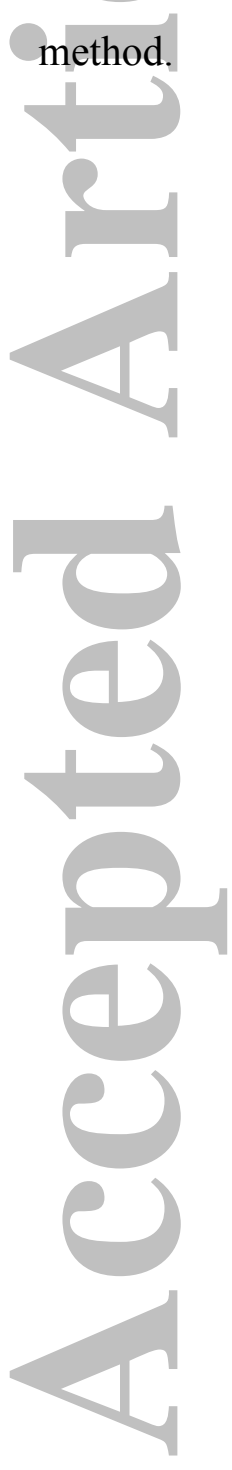


\section{Acknowledgements}

This work was previously presented as a poster in the 2012 Radiological Society of North America meeting in Chicago, IL.
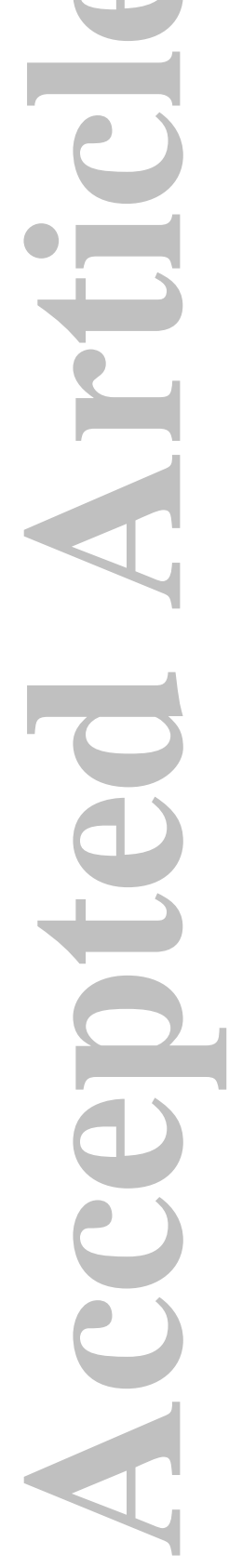


\section{References}

1. Nowygrod R, Egorova N, Greco G, et al. Trends, complications, and mortality in peripheral vascular surgery. J Vasc Surg 2006; 43:205-216.

2. Vikatmaa P, Mitchell D, Jensen LP, et al. Variation in clinical practice in carotid surgery in nine countries 2005-2010. Lessons from VASCUNET and recommendations for the future of national clinical audit. Eur J Vasc Endovasc Surg 2012; 44:11-17.

3. Dumont TM, Rughani AI. National trends in carotid artery revascularization surgery. J Neurosurg 2012; 116:1251-1257.

4. Alqadri SL, Qureshi AI. Treatment of symptomatic carotid stenosis: carotid stent placement versus endarterectomy. Curr Atheroscler Rep 2013; 15:345.

5. Buschur M, Gurm HS. Carotid artery stenting versus carotid endarterectomy: post CREST. Curr Cardiol Rep. 2012; 14:135-141.

6. Weinstein S, Mabray MC, Aslam R, Hope T, Yee J, Owens C. Intraoperative sonography during carotid endarterectomy. Normal appearance and spectrum of complications. J Ultrasound Med 2015; 34:885-894.

7. Ascher E, Markevich N, Kallakuri S, Schutzer RW, Hingorani AP. Intraoperative carotid artery duplex scanning in a modern series of 650 consecutive primary endarterectomy procedures. J Vasc Surg 2004; 39:416-420.

8. Patel RR, Adam R, Maldjian C, Lincoln CM, Yuen A, Arneja A. Cervical carotid artery dissection: current review of diagnosis and treatment. Cardiol Rev 2012; $20: 145-152$. 
9. Schievink WI, Mokri B, Whisnant JP. Internal carotid artery dissection in a community. Rochester, Minnesota, 1987-1992. Stroke 1993; 24:1678-80.

10. Giroud M, Fayolle H, Andre N, et al. Incidence of internal carotid artery dissection in the community of Dijon. J Neurol Neurosurg Psychiatry 1994; $57: 1443$.

11. Paramasivam S, Leesch W, Fifi J, Ortiz R, Niimi Y, Berenstein A. Iatrogenic (1) dissection during neurointerventional procedures: a retrospective analysis. $J$ Neurointerv Surg 2012; 4:331-335.

12. Inamasu J, Guiot BH. Iatrogenic carotid artery injury in neurosurgery. Neurosurg Rev 2005; 28:239-247.

13. Kim YK, Schulman S. Cervical artery dissection: pathology, epidemiology and management. Thromb Res 2009; 123:810-821.

14. Flis CM, Jager HR, Sidhu PS. Carotid and vertebral artery dissections: clinical aspects, imaging features and endovascular treatment. Eur Radiol 2007; 17:820834.

15. Grau AJ, Brandt T, Buggle F, et al. Association of cervical artery dissection with recent infection. Arch Neurol 1999;56:851-856.

16. Schievink WI. Spontaneous dissection of the carotid and vertebral arteries. $N E n g l$ $J$ Med 2001; 344:898-906.

17. Self DD, Bryson GL, Sullivan PJ. Risk factors for post-carotid endarterectomy hematoma formation. Can J Anaesth 1999; 46:635-640. 
18. Zinkstok SM, Vergouwen MD, Engelter ST, et al. Safety and functional outcome of thrombolysis in dissection-related ischemic stroke: a meta-analysis of individual patient data. Stroke 2011; 42:2515-2520.

19. Lyrer P, Engelter S. Antithrombotic drugs for carotid artery dissection. Cochrane Database Syst Rev 2010:Cd000255.

20. Yaghi S, Maalouf N, Keyrouz SG. Cervical artery dissection: risk factors, treatment, and outcome; a 5-year experience from a tertiary care center. Int J Neurosci 2012; 122:40-44.

21. Millet JD, Stilp E, Kirsch JD, et al. Carotid stent deformation: Sonographic findings and CT correlation. J Clin Ultrasound 2014; 42:313-317.

22. Ferguson GG, Eliasziw M, Barr HW, et al. The North American Symptomatic Carotid Endarterectomy Trial: surgical results in 1415 patients. Stroke 1999; $30: 1751-1758$.

23. Comerota AJ, Difiore R, Tzilinis A, Chahwan S. Cervical hematoma following carotid endarterectomy is morbid and preventable: a 12-year case-controlled review. Vasc Endovascular Surg 2012;46: 610-616.

24. Grandhi R, Gande A, Zwagerman NT, Jankowitz BT. Facial and neck hematoma after carotid artery stenting: an uncommon misadventure in endovascular carotid revascularization. J Neurointerv Surg 2014; 6:e39.

25. Liu ZJ, Fu WG, Guo ZY, Shen LG, Shi ZY, Li JH. Updated systematic review and meta-analysis of randomized clinical trials comparing carotid artery stenting and carotid endarterectomy in the treatment of carotid stenosis. Ann Vasc Surg $2012 ; 26: 576-590$. 
26. Stone DH, Nolan BW, Schanzer A, et al. Protamine reduces bleeding complications associated with carotid endarterectomy without increasing the risk of stroke. J Vasc Surg 2010; 51:559-564.

27. Chiesa R, Melissano G, Castellano R, Frigerio S, Catenaccio B. Carotid endarterectomy: experience in 5425 cases. Ann Vasc Surg 2004;18:527-534.

28. Naughton PA, Garcia-Toca M, Rodriguez HE, Pearce WH, Eskandari MK, Morasch MD. Carotid artery reconstruction for infected carotid patches. Eur J Vasc Endovasc Surg 2010; 40:492-498.

29. Naylor AR, Payne D, London NJ, et al. Prosthetic patch infection after carotid endarterectomy. Eur J Vasc Endovasc Surg 2002; 23:11-16.

30. Knight BC, Tait WF. Dacron patch infection following carotid endarterectomy: a systematic review of the literature. Eur J Vasc Endovasc Surg 2009; 37:140-148.

31. Kalapatapu VR, Shelton KR, Ali AT, Moursi MM, Eidt JF. Pseudoaneurysm: a review. Curr Treat Options Cardiovasc Med 2008; 10:173-183.

32. Abdelhamid MF, Wall ML, Vohra RK. Carotid artery pseudoaneurysm after carotid endarterectomy: case series and a review of the literature. Vasc Endovascular Surg 2009; 43:571-577.

33. Stirling MJ, Smith TP, McCann RL. Carotid artery angioplasty and stent placement: postprocedural hemorrhage and delayed pseudoaneurysm formation. $J$ Vasc Interv Radiol 2008; 19:595-599.

34. Guilbert MC, Elkouri S, Bracco D, et al. Arterial trauma during central venous catheter insertion: Case series, review and proposed algorithm. J Vasc Surg 2008; 48:918-925. 
35. Garg K, Rockman CB, Lee V, et al. Presentation and management of carotid artery aneurysms and pseudoaneurysms. J Vasc Surg 2012; 55:1618-1622.

36. Defillo A, Zelensky A, Pulivarthi S, et al. Non-infected carotid artery pseudoaneurysm 29 years after endarterectomy, endovascular management with covered stent. J Neurosurg Sci 2012; 56:145-149.

37. Li Z, Chang G, Yao C, et al. Endovascular stenting of extracranial carotid artery aneurysm: a systematic review. Eur J Vasc Endovasc Surg 2011; 42:419-426.

38. Al-Damluji MS, Nagpal S, Stilp E, Remetz M, Mena C. Carotid revascularization: a systematic review of the evidence. J Interv Cardiol 2013; 26:399-410.

39. Brott TG, Hobson RW, 2nd, Howard G, et al. Stenting versus endarterectomy for treatment of carotid-artery stenosis. $N$ Engl J Med 2010; 363:11-23.

40. Mas JL, Chatellier G, Beyssen B, et al. Endarterectomy versus stenting in patients with symptomatic severe carotid stenosis. $N$ Engl J Med 2006; 355:1660-1671.

41. Bonati LH, Dobson J, Featherstone RL, et al. Long-term outcomes after stenting versus endarterectomy for treatment of symptomatic carotid stenosis: the International Carotid Stenting Study (ICSS) randomised trial. Lancet 2015; 385:529-538.

42. Owens EL, Kumins NH, Bergan JJ, Sparks SR. Surgical management of acute complications and critical restenosis following carotid artery stenting. Ann Vasc Surg 2002; 16:168-75.

43. Markatis F, Petrosyan A, Abdulamit T, Bergeron P. Acute carotid stent thrombosis: a case of surgical revascularization and review of treatment options. Vascular 2012; 20:217-220. 
44. den Hartog AG, Algra A, Moll FL, de Borst GJ. Mechanisms of gender-related outcome differences after carotid endarterectomy. J Vasc Surg 2010; 52:10621071.

45. Halm EA, Tuhrim S, Wang JJ, Rockman C, Riles TS, Chassin MR. Risk factors for perioperative death and stroke after carotid endarterectomy: results of the new york carotid artery surgery study. Stroke 2009; 40:221-229.

46. Khan M, Qureshi AI. Factors associated with increased rates of post-procedural stroke or death following carotid artery stent placement: a systematic review. $J$ Vasc Interv Neurol 2014; 7:11-20.

47. Lal BK, Beach KW, Roubin GS, et al. Restenosis after carotid artery stenting and endarterectomy: a secondary analysis of CREST, a randomised controlled trial. Lancet Neurol 2012; 11:755-763.

48. Eckstein HH, Ringleb P, Allenberg JR, et al. Results of the stent-protected angioplasty versus carotid endarterectomy (SPACE) study to treat symptomatic stenoses at 2 years: a multinational, prospective, randomised trial. Lancet Neurol $2008 ; 7: 893-902$.

49. Setacci F, Sirignano P, Galzerano G, de Donato G, Cappelli A, Setacci C. Carotid restenosis after endarterectomy and stenting: a critical issue? Ann Vasc Surg $2013 ; 27: 888-893$.

50. Barros P, Felgueiras H, Pinheiro D, Guerra M, Gama V, Veloso M. Restenosis after carotid artery stenting using a specific designed ultrasonographic protocol. $J$ Stroke Cerebrovasc Dis 2014; 23:1416-1420. 
51. Lal BK. Recurrent carotid stenosis after CEA and CAS: diagnosis and management. Semin Vasc Surg 2007; 20:259-266.

52. Reina-Gutierrez T, Serrano-Hernando FJ, Sanchez-Hervas L, Ponce A, Vega de Ceniga M, Martin A. Recurrent carotid artery stenosis following endarterectomy: natural history and risk factors. Eur J Vasc Endovasc Surg 2005; 29:334-341.

53. Liapis CD, Kakisis JD, Kostakis AG. Recurrent carotid artery stenosis: natural history and predisposing factors. A long-term follow-up study. Int Angiol 2001; 20:330-336.

54. Van Laanen J, Hendriks JM, Van Sambeek MR. Factors influencing restenosis after carotid artery stenting. J Cardiovasc Surg (Torino) 2008; 49:743-747.

55. Bekelis K, Moses Z, Missios S, Desai A, Labropoulos N. Indications for treatment of recurrent carotid stenosis. Br J Surg 2013; 100:440-447.

56. Attigah N, Kulkens S, Deyle C, et al. Redo surgery or carotid stenting for restenosis after carotid endarterectomy: results of two different treatment strategies. Ann Vasc Surg 2010; 24:190-195.

57. van Haaften AC, Bots ML, Moll FL, de Borst GJ. Therapeutic options for carotid in-stent restenosis: review of the literature. J Vasc Interv Radiol 2010; 21:14711477.

58. Grant EG, Benson CB, Moneta GL, et al. Carotid artery stenosis: gray-scale and Doppler US diagnosis--Society of Radiologists in Ultrasound consensus conference. Radiology 2003; 229:340-346.

59. Setacci C, Chisci E, Setacci F, Iacoponi F, de Donato G. Grading carotid intrastent restenosis: a 6-year follow-up study. Stroke 2008; 39:1189-1196. 
60. Armstrong PA, Bandyk DF, Johnson BL, et al. Duplex scan surveillance after carotid angioplasty and stenting: a rational definition of stent stenosis. J Vasc Surg 2007;46:460-465.

61. Zhou W, Felkai DD, Evans M, et al. Ultrasound criteria for severe in-stent restenosis following carotid artery stenting. J Vasc Surg 2008; 47:74-80.

62. Chi YW, White CJ, Woods TC, Goldman CK. Ultrasound velocity criteria for carotid in-stent restenosis. Catheter Cardiovasc Interv 2007; 69:349-354.

63. Garcia-Toca M, Rodriguez HE, Naughton PA, et al. Are carotid stent fractures clinically significant? Cardiovasc Intervent Radiol 2012; 35:263-267.

64. Chang CK, Huded CP, Nolan BW, Powell RJ. Prevalence and clinical significance of stent fracture and deformation following carotid artery stenting. $J$ Vasc Surg 2011; 54:685-690.

65. Ling AJ, Mwipatayi P, Gandhi T, Sieunarine K. Stenting for carotid artery stenosis: fractures, proposed etiology and the need for surveillance. J Vasc Surg $2008 ; 47: 1220-1226$.

66. Coppi G, Moratto R, Veronesi J, Nicolosi E, Silingardi R. Carotid artery stent fracture identification and clinical relevance. J Vasc Surg 2010; 51:1397-1405.

67. Bakar B, Cekirge S, Tekkok IH. External carotid-internal jugular fistula as a late complication after carotid endarterectomy: a rare case. Cardiovasc Intervent Radiol 2011; 34(2): S53-S56. 


\section{Tables}

Table 1. Classification of complications following carotid artery interventions

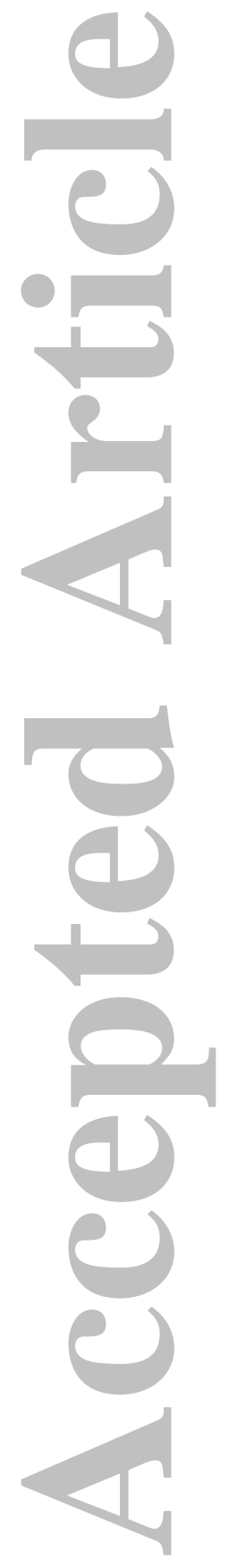

\begin{tabular}{|c|c|}
\hline Intra procedural & Post-procedural \\
\hline \multirow[t]{7}{*}{ Dissection } & $\begin{array}{l}\text { Fluid collection } \\
\text { - Hematoma } \\
\text { - Abscess } \\
\text { - Suture dehiscence }\end{array}$ \\
\hline & Pseudoaneurysm \\
\hline & Thrombosis \\
\hline & Thromboembolism \\
\hline & Restenosis \\
\hline & Stent deformation or fracture \\
\hline & Arteriovenous fistula \\
\hline
\end{tabular}




\section{Figure Legends}

Figure 1. 84 year old woman, status post right CEA complicated by a difficult surgical reconstruction. A, Intraoperative sagittal grey scale US demonstrates a small intimal flap in the right carotid bulb (arrow). B, Smaller intimal flaps, adherent fibrin strands, or thrombi are also seen along the anterior and posterior walls of the superior aspect of the (1) endarterectomy site (arrows). Given the small size of the intimal flap, the patient was managed medically with $325 \mathrm{mg}$ /day aspirin. She had an uneventful postoperative course and remained asymptomatic at 3 months follow-up.

Figure 2. 65 year old man who underwent left CEA one day previously and developed bleeding from the incision site. A and $\mathbf{B}$, Sagittal grey scale and transverse color Doppler US of the neck show a large collection with a fluid-fluid level, consistent with an acute hematoma, presumably secondary to suture dehiscence. He developed a stroke soon afterwards. C, CT scan of the head without contrast demonstrates a left posterior circulation infarct (arrow).

Figure 3. 69 year old man who presented with a non-pulsatile left neck mass following left CEA. A and B, Sagittal and transverse color Doppler US demonstrate an anechoic fluid collection adjacent to the left CCA and ICA, measuring $2.0 \times 2.1 \mathrm{~cm}$ in size. C, The left CCA is patent (arrow). Findings are consistent with a post-operative hematoma versus seroma. 
Figure 4. 81 year old man, status post left CEA with bovine patch angioplasty 1 week prior, who presented with left neck pain. Transverse grey scale US of the left neck demonstrates a poorly defined area of heterogeneous echogenicity measuring $5.1 \times 2.0 \times$ $4.3 \mathrm{~cm}$, outlined with calipers. Note the punctate echogenic foci (arrows) within the collection that likely represent gas bubbles. While this appearance may be seen with both abscess and hematoma (secondary to aseptic breakdown of blood products), in this case, the collection resolved without intervention.

Figure 5. 83 year old woman status post left CEA three years previously who presented with a hard, enlarging left neck mass. A, Sagittal grey scale US of the left CCA demonstrates an outpouching, consistent with a wide-neck pseudoaneurysm. Note internal echoes (arrow) that fill in approximately half of the pseudoaneurysm lumen, consistent with partial thrombosis. B, A "yin-yang" pattern of swirling blood flow was observed within the residual lumen on color Doppler interrogation. C, Angiography confirmed the presence of a CCA pseudoaneurysm (arrow).

Figure 6. 60 year old man who experienced a transient ischemic attack a few hours following an uneventful CAS of the left ICA. A and B, Transverse color and sagittal spectral Doppler US of the left ICA show a string sign (arrow in A) and an increased velocity of $273 \mathrm{~cm} / \mathrm{s}$ within the stent. He was subsequently discharged to home on dual antiplatelet therapy. On post procedure day 5, he presented to the emergency room with aphasia. $\mathbf{C}$ and D, Repeat sagittal color and spectral Doppler US of the left ICA show no detectable flow within the stent, concerning for complete thrombosis. E, Axial diffusion 
weighted MRI of the brain demonstrates multiple foci of increased signal in the left cerebral hemisphere. F, These foci are hypointense on the corresponding apparent diffusion coefficient map, consistent with a thromboembolic stroke. G, CTA of the neck performed a few months later for evaluation of the right side redemonstrates the known left ICA stent occlusion (arrow).

Figure 7. 82 year old woman with prior surgical history of left CEA, who developed left sided weakness, left facial droop, and dysarthria 4 hours following right CEA. A There is no demonstrable flow within the right distal CCA on sagittal color Doppler US, suggestive of complete thrombosis. B, Axial diffusion weighted MRI of the brain demonstrates increased signal in portions of the right parietal and frontal lobes, consistent with an acute infarction in the territory of the right middle cerebral artery.

Figure 8. 65 year old man who is status post left CEA 9 years previously. A and B, Sagittal color and spectral Doppler US demonstrate color aliasing with an increased flow velocity of $234 \mathrm{~cm} / \mathrm{s}$ within the left proximal ICA, concerning for a high grade stenosis. C, DSA confirms a high-grade focal left proximal ICA stenosis (arrow). The patient subsequently underwent a technically successful CAS (not shown). D and E, Sagittal color and spectral Doppler US 1 month later demonstrate a widely patent stent with a normal peak systolic velocity of $106 \mathrm{~cm} / \mathrm{s}$. F, Repeat angiogram 2 years later shows highgrade focal in-stent restenosis (arrow). The patient was treated with angioplasty (not shown). 
Figure 9. 73 year old man with history of prior strokes, and who had also received radiation therapy for throat cancer. A, Sagittal spectral Doppler US demonstrates a highgrade stenosis within the left distal CCA with a peak systolic velocity of $469 \mathrm{~cm} / \mathrm{s}$. B, Severe CCA stenosis is confirmed on 3D reconstructed CTA (arrow). C, The stenosis is also demonstrated on DSA (arrow). The patient underwent successful CAS with angioplasty. D and E, Post-stent angiogram demonstrates a focal bulge in the location of the prior stenosis (arrow), consistent with stent deformation without evidence of a fracture. A few days following the procedure, the patient had a transient ischemic attack. F, Grey scale US demonstrates the mildy deformed but intact stent struts. G, Sagittal color and spectral Doppler US of the proximal left ICA demonstrates interval appearance of a hypoechoic area at the origin of the left ICA (arrow) with an elevated peak systolic velocity of $191 \mathrm{~cm} / \mathrm{s}$ (scale not shown), consistent with an acute thrombus. H, Diffusion weighted MRI of the brain shows multiple hyperintense foci confined to the left cerebral hemisphere, consistent with multiple thromboemboli from a carotid source. 


\section{Abbreviations}

CAS, Carotid artery stenting; CCA, Common carotid artery; CEA, Carotid endarterectomy; CT, Computed tomography; CTA, Computed tomography angiography; DSA, Digital subtraction angiography; EDV, End diastolic velocity; ICA, Internal carotid artery; PSA, Pseudoaneurysm; PSV, Peak systolic velocity; MRA, Magnetic resonance angiography; MRI, Magnetic resonance imaging; US, Ultrasound
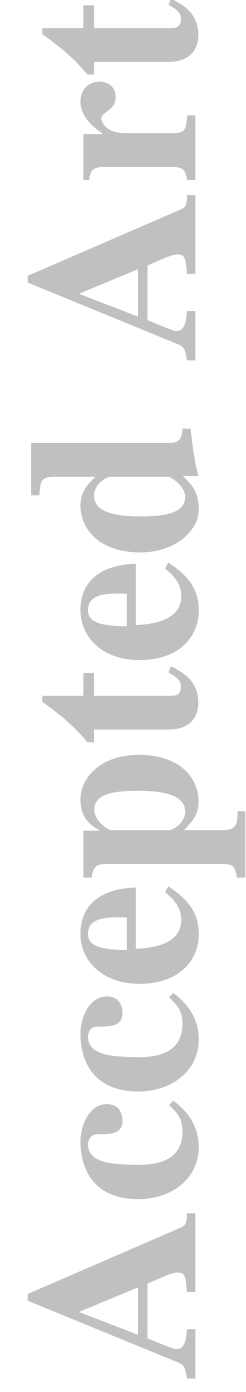


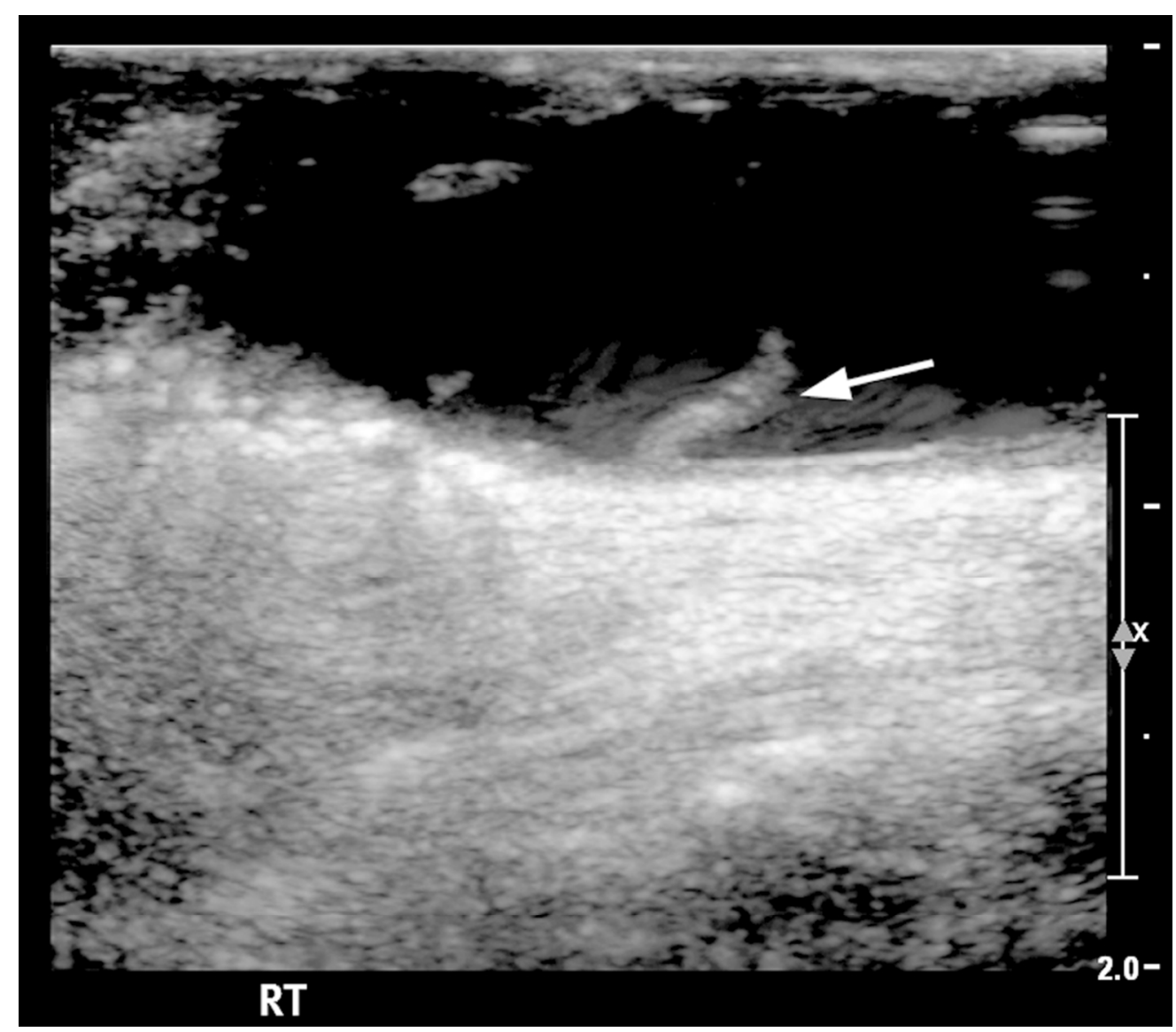

Figure 1A. 84 year old woman, status post right CEA complicated by a difficult surgical reconstruction. A, Intraoperative sagittal grey scale US demonstrates a small intimal flap in the right carotid bulb (arrow). B, Smaller intimal flaps, adherent fibrin strands, or thrombi are also seen along the anterior and posterior walls of the superior aspect of the endarterectomy site (arrows). Given the small size of the intimal flap, the patient was managed medically with $325 \mathrm{mg} /$ day aspirin. She had an uneventful postoperative course and remained asymptomatic at 3 months follow-up.

$79 \times 69 \mathrm{~mm}(300 \times 300 \mathrm{DPI})$

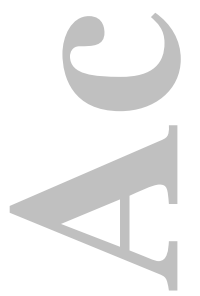




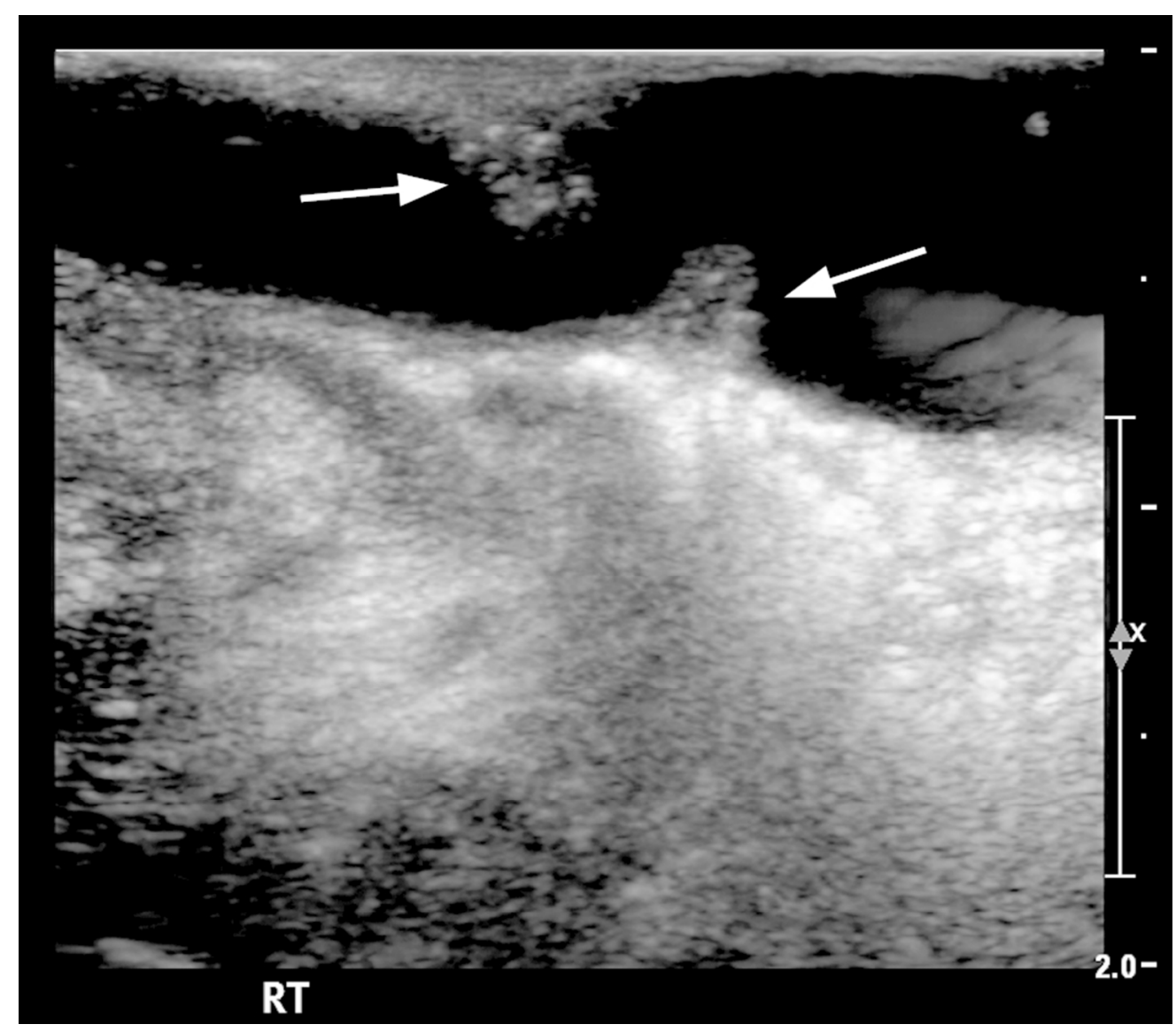

Figure 1B. 84 year old woman, status post right CEA complicated by a difficult surgical reconstruction. A, Intraoperative sagittal grey scale US demonstrates a small intimal flap in the right carotid bulb (arrow). B, Smaller intimal flaps, adherent fibrin strands, or thrombi are also seen along the anterior and posterior walls

of the superior aspect of the endarterectomy site (arrows). Given the small size of the intimal flap, the patient was managed medically with $325 \mathrm{mg} /$ day aspirin. She had an uneventful postoperative course and remained asymptomatic at 3 months follow-up.

$79 \times 69 \mathrm{~mm}(300 \times 300$ DPI)

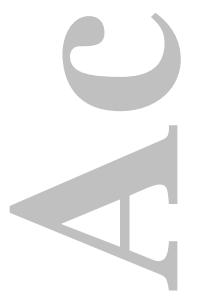




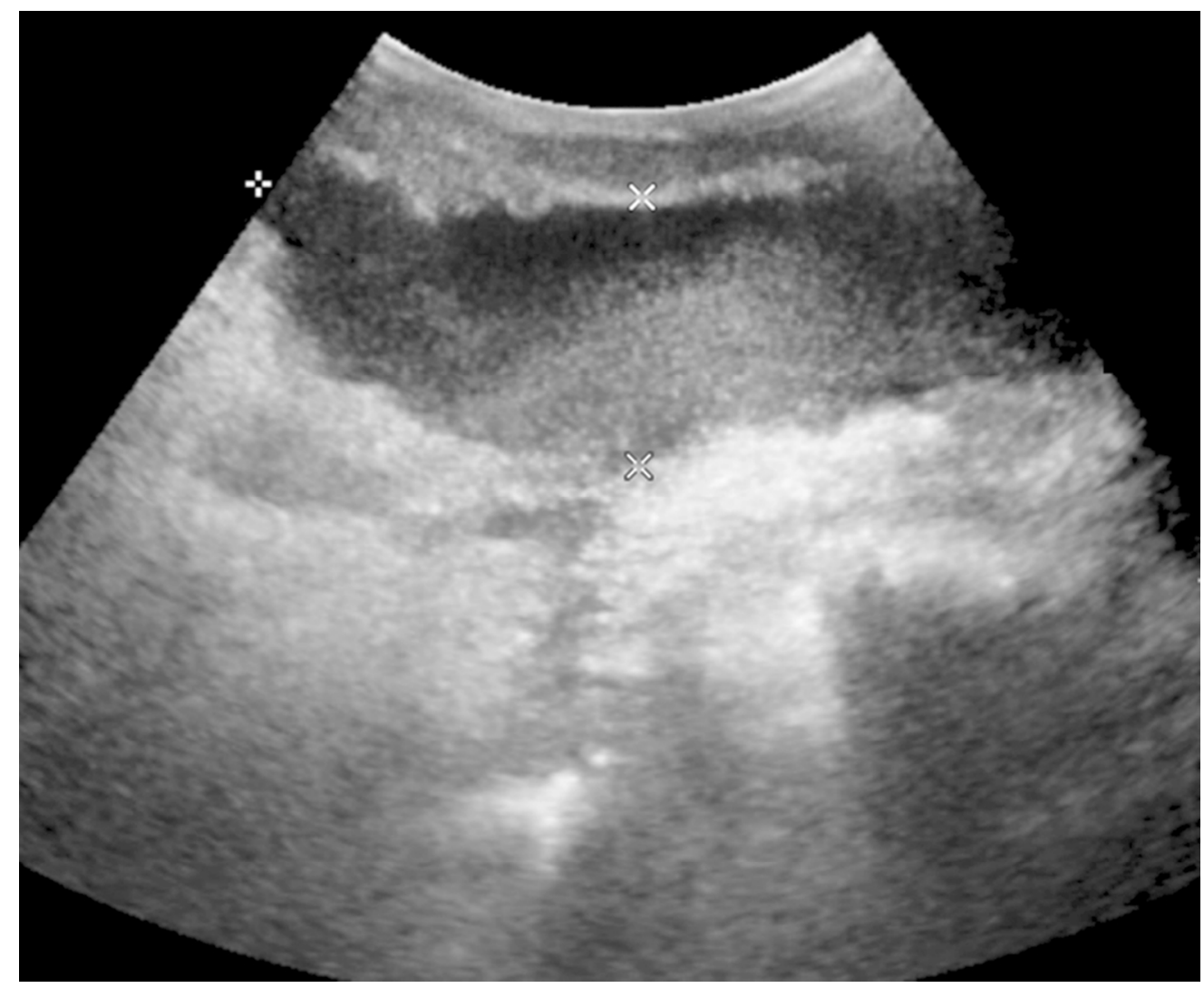

Figure 2A. 65 year old man who underwent left CEA one day previously and developed bleeding from the incision site. A and B, Sagittal grey scale and transverse color Doppler US of the neck show a large collection with a fluid-fluid level, consistent with an acute hematoma, presumably secondary to suture dehiscence. He developed a stroke soon afterwards. C, CT scan of the head without contrast demonstrates a left posterior circulation infarct (arrow).

$79 \times 65 \mathrm{~mm}(300 \times 300 \mathrm{DPI})$ 


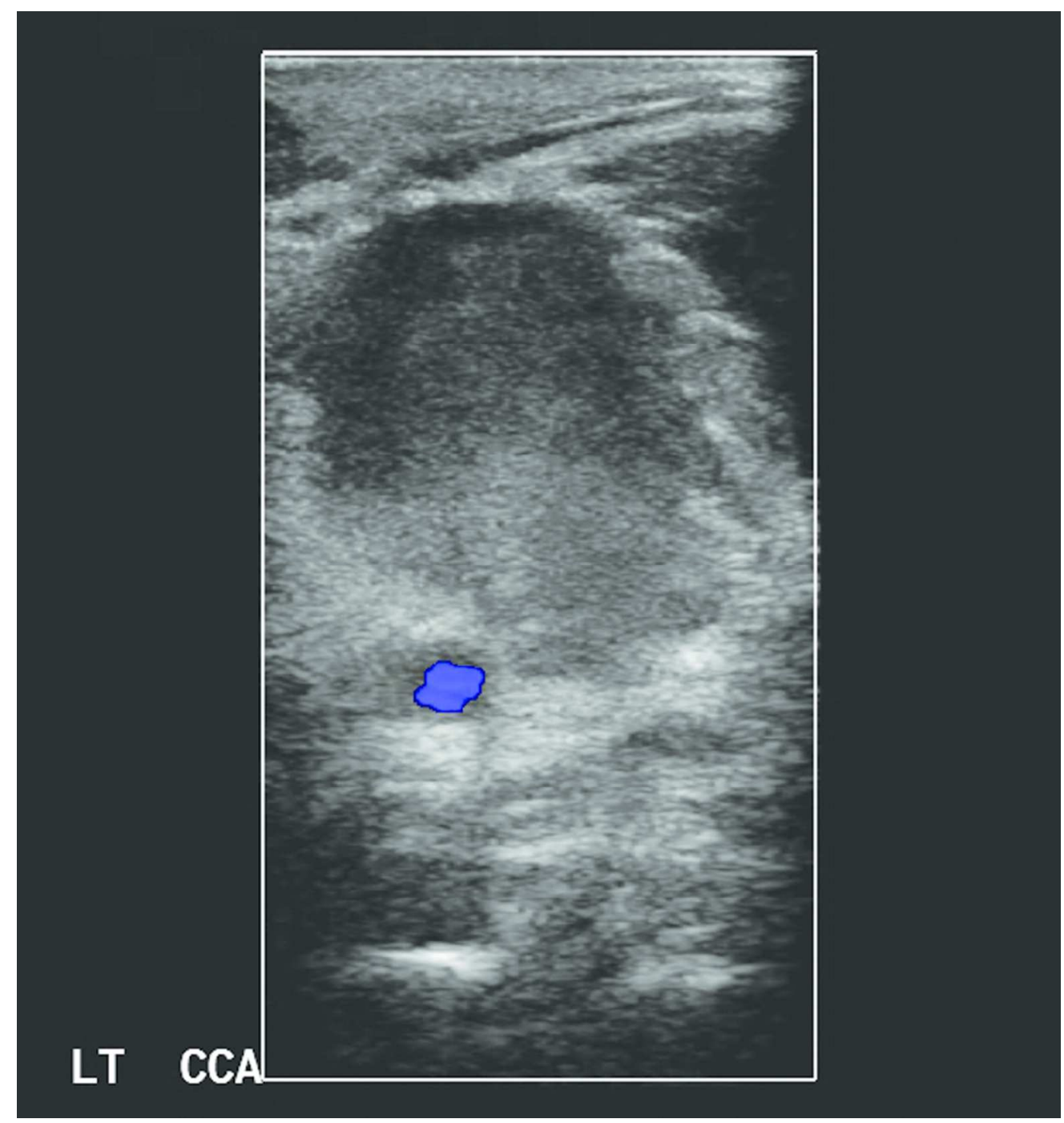

Figure 2B. 65 year old man who underwent left CEA one day previously and developed bleeding from the incision site. A and B, Sagittal grey scale and transverse color Doppler US of the neck show a large collection with a fluid-fluid level, consistent with an acute hematoma, presumably secondary to suture dehiscence. He developed a stroke soon afterwards. C, CT scan of the head without contrast demonstrates a left posterior circulation infarct (arrow).

$79 \times 84 \mathrm{~mm}(300 \times 300$ DPI $)$ 


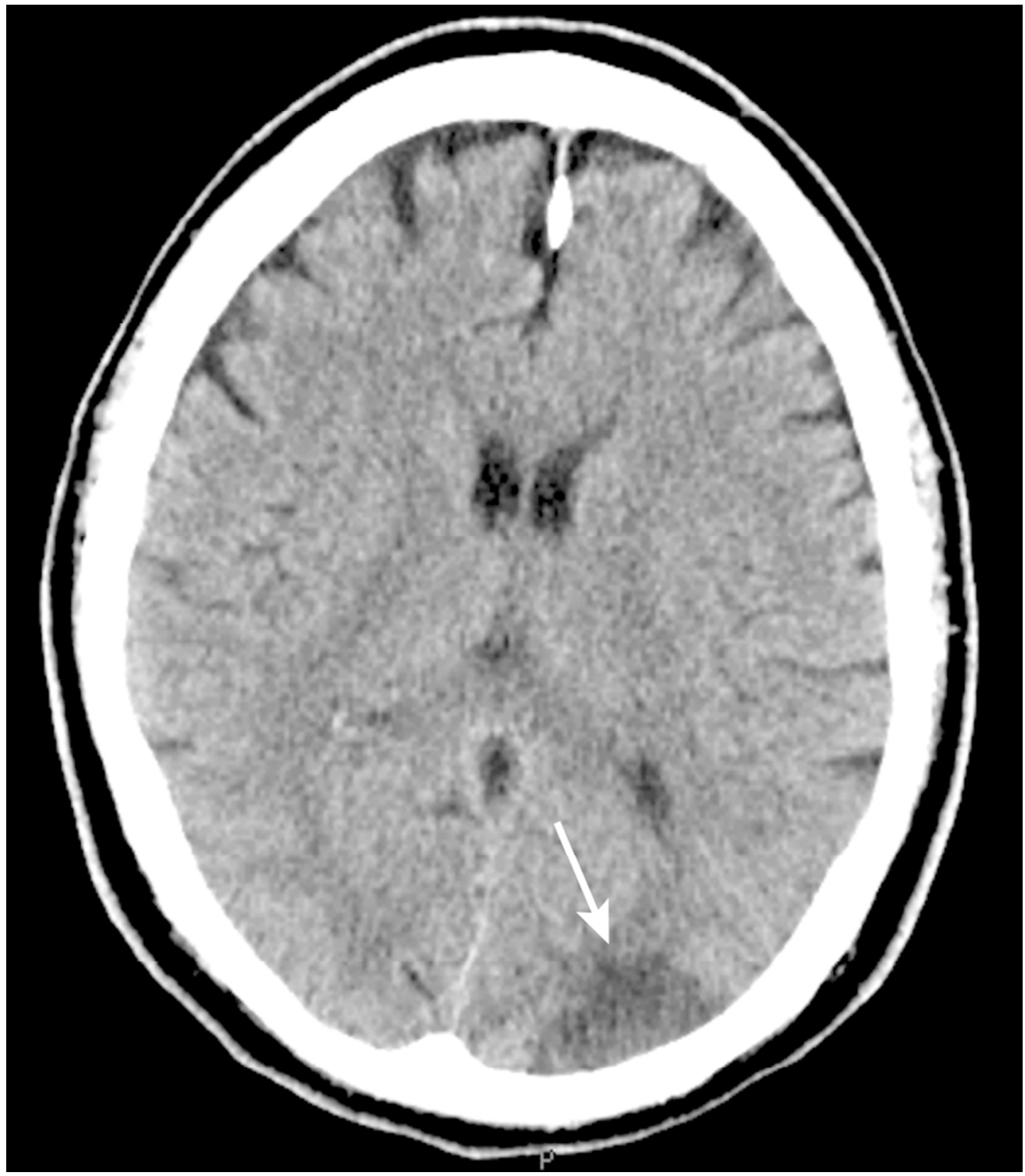

Figure 2C. 65 year old man who underwent left CEA one day previously and developed bleeding from the incision site. A and B, Sagittal grey scale and transverse color Doppler US of the neck show a large collection with a fluid-fluid level, consistent with an acute hematoma, presumably secondary to suture dehiscence. He developed a stroke soon afterwards. C, CT scan of the head without contrast demonstrates a left posterior circulation infarct (arrow).

$79 \times 91 \mathrm{~mm}(300 \times 300 \mathrm{DPI})$ 


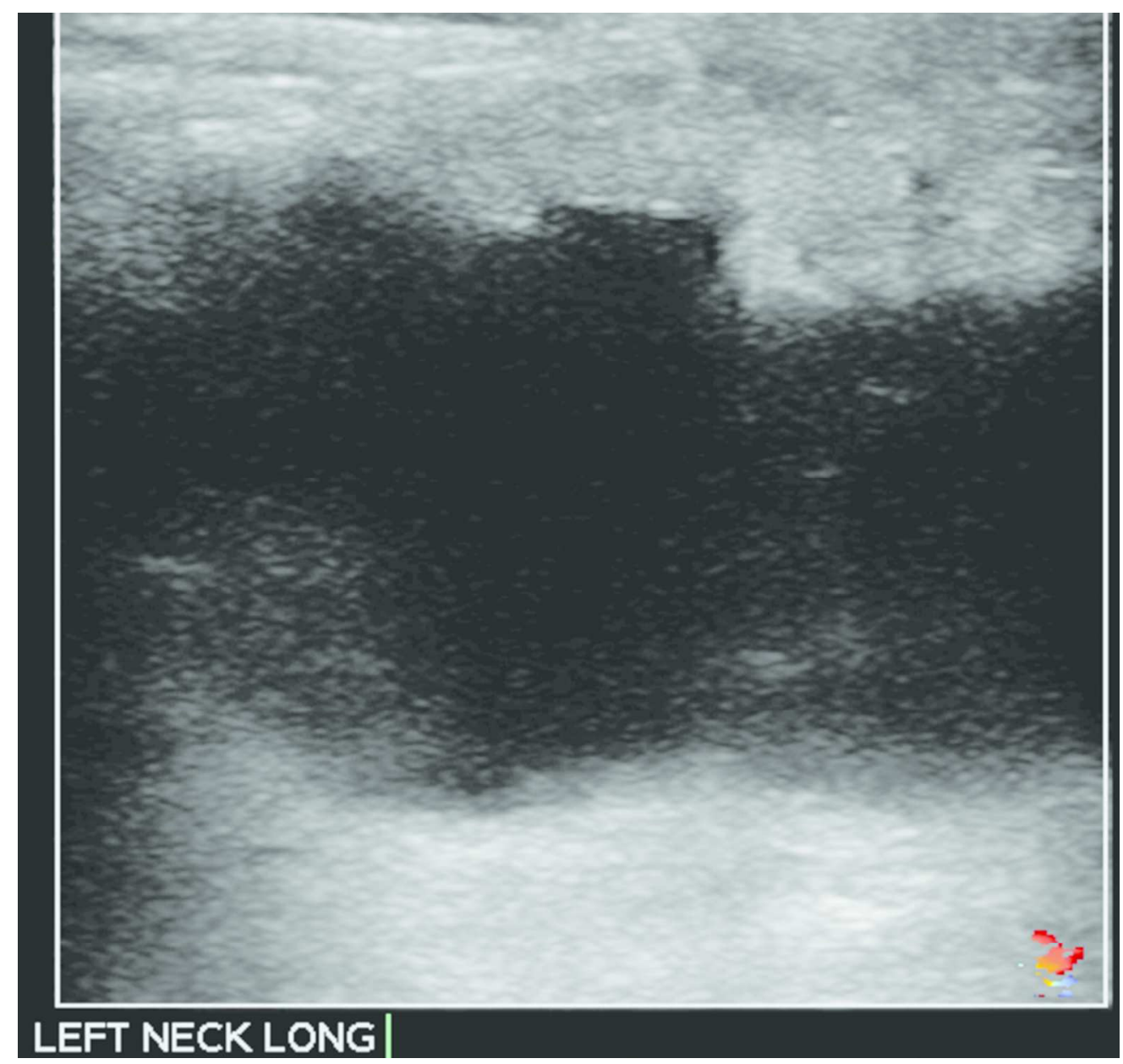

Figure 3A. 69 year old man who presented with a non-pulsatile left neck mass following left CEA. A and B, Sagittal and transverse color Doppler US demonstrate an anechoic fluid collection adjacent to the left CCA and ICA, measuring $2.0 \times 2.1 \mathrm{~cm}$ in size. C, The left CCA is patent (arrow). Findings are consistent with a post-operative hematoma versus seroma.

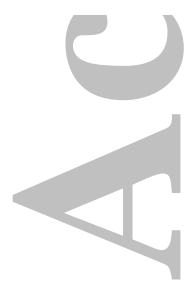

$79 \times 75 \mathrm{~mm}(300 \times 300$ DPI $)$ 


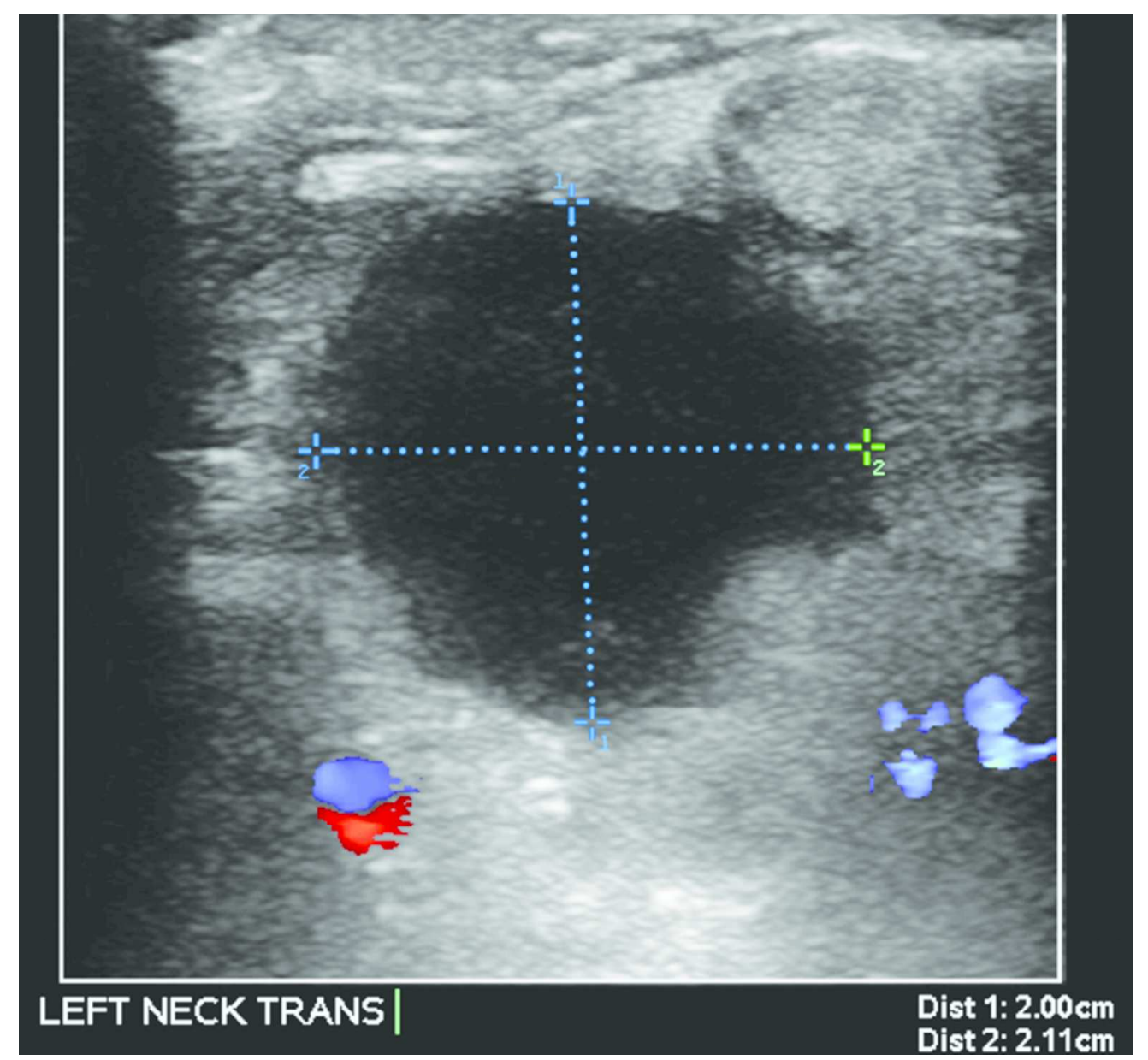

Figure 3B. 69 year old man who presented with a non-pulsatile left neck mass following left CEA. A and B, Sagittal and transverse color Doppler US demonstrate an anechoic fluid collection adjacent to the left CCA and ICA, measuring $2.0 \times 2.1 \mathrm{~cm}$ in size. C, The left CCA is patent (arrow). Findings are consistent with a post-operative hematoma versus seroma.

$79 \times 74 \mathrm{~mm}(300 \times 300$ DPI $)$

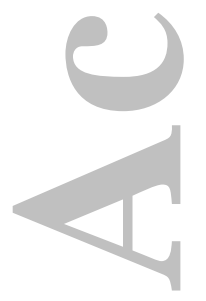




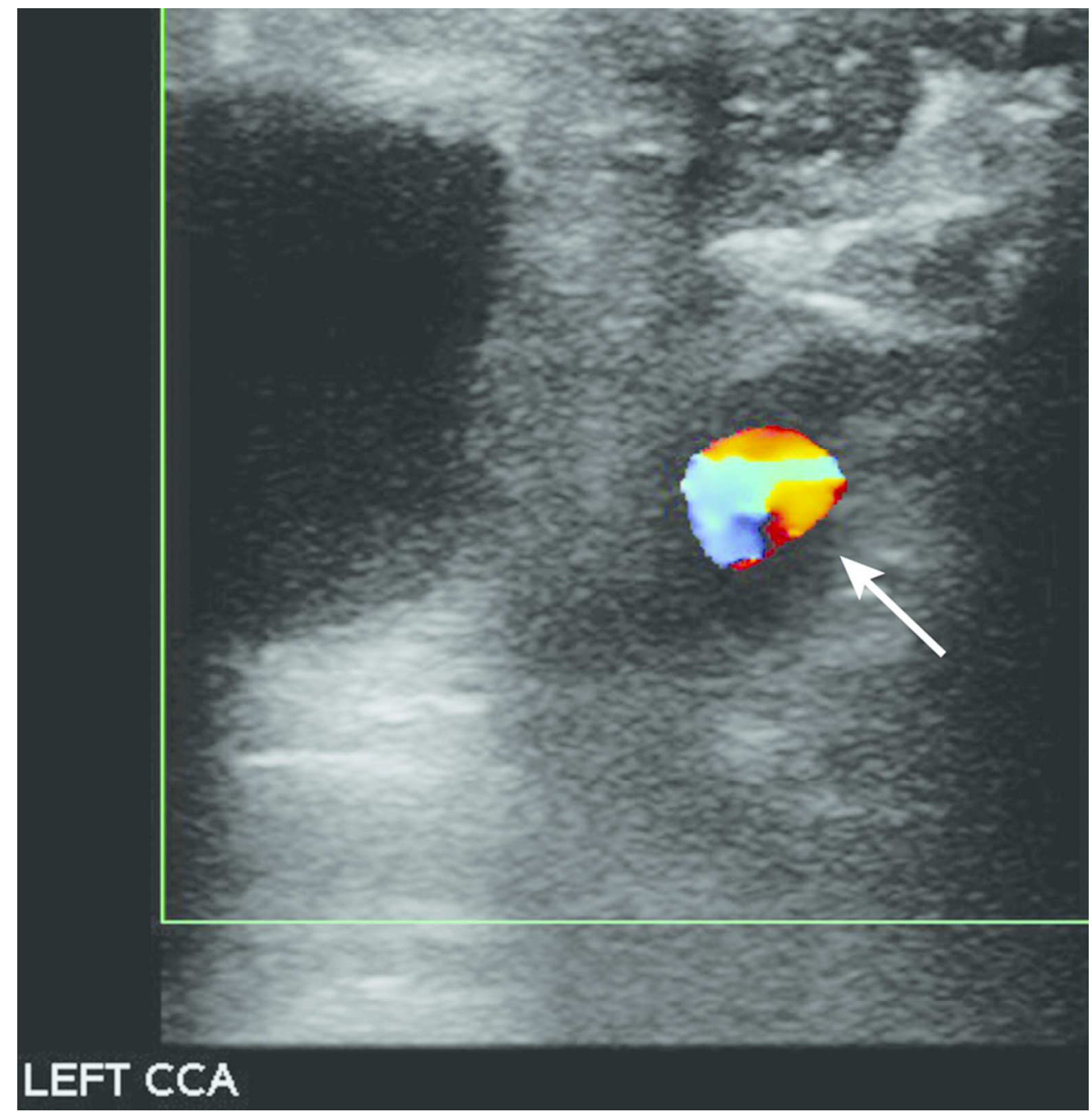

Figure 3C. 69 year old man who presented with a non-pulsatile left neck mass following left CEA. A and B, Sagittal and transverse color Doppler US demonstrate an anechoic fluid collection adjacent to the left CCA and ICA, measuring $2.0 \times 2.1 \mathrm{~cm}$ in size. C, The left CCA is patent (arrow). Findings are consistent with a post-operative hematoma versus seroma.

$79 \times 81 \mathrm{~mm}(300 \times 300 \mathrm{DPI})$

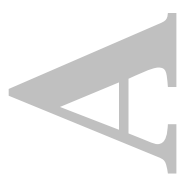




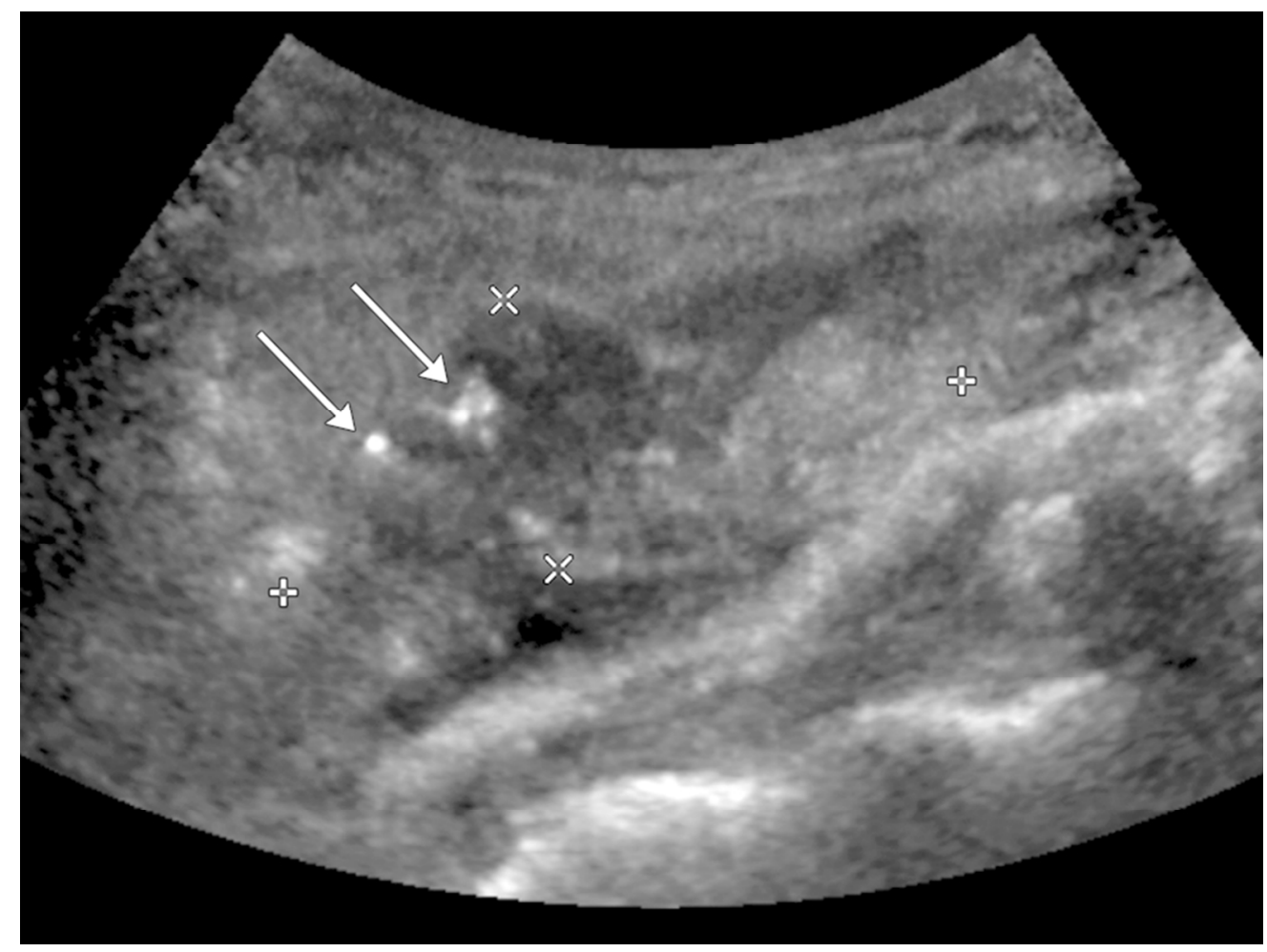

Figure 4.81 year old man, status post left CEA with bovine patch angioplasty 1 week prior, who presented with left neck pain. Transverse grey scale US of the left neck demonstrates a poorly defined area of heterogeneous echogenicity measuring $5.1 \times 2.0 \times 4.3 \mathrm{~cm}$, outlined with calipers. Note the punctate echogenic foci (arrows) within the collection that likely represent gas bubbles. While this appearance may be seen with both abscess and hematoma (secondary to aseptic breakdown of blood products), in this case, the collection resolved without intervention.

$79 \times 59 \mathrm{~mm}(300 \times 300 \mathrm{DPI})$ 


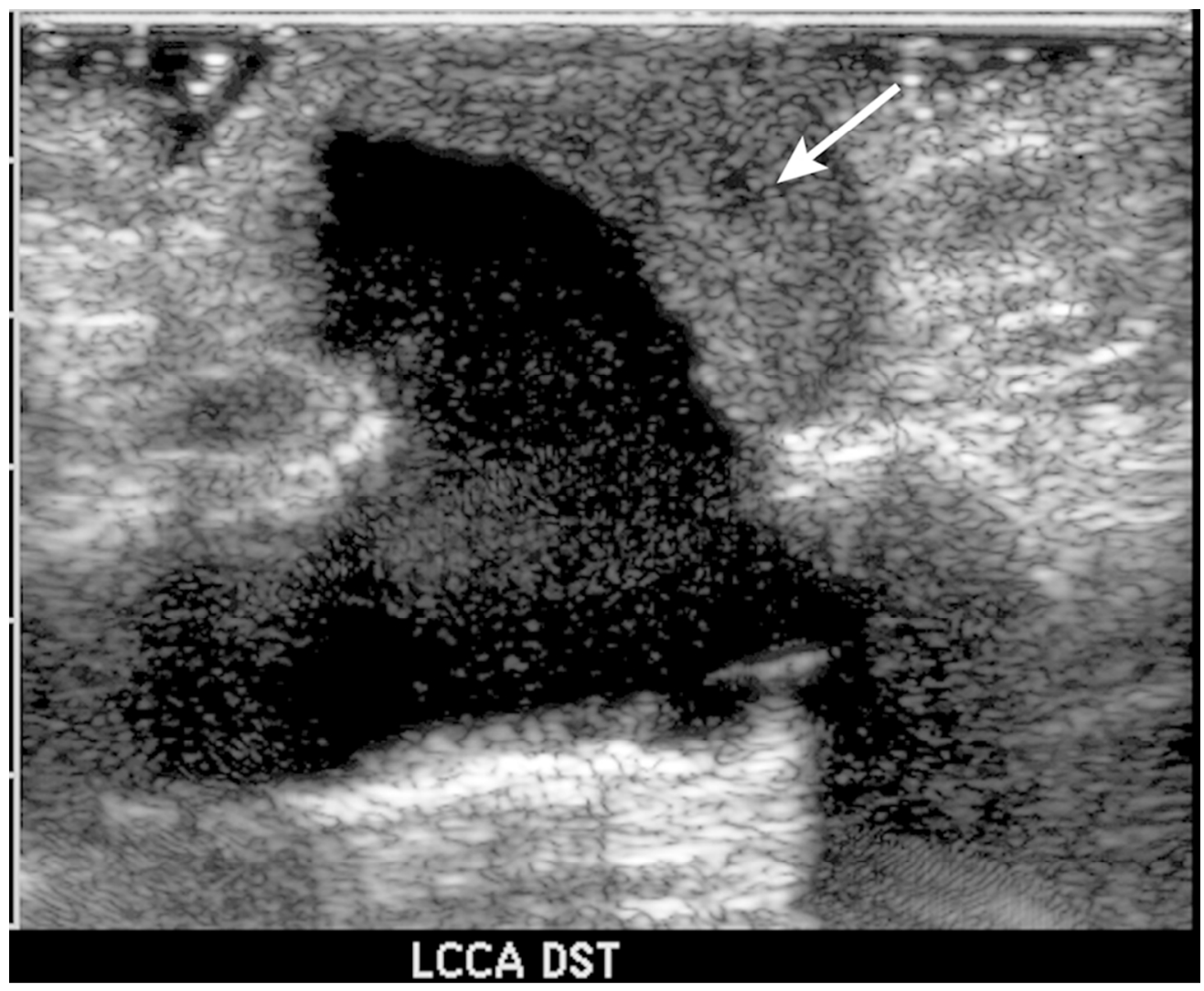

Figure 5A. 83 year old woman status post left CEA three years previously who presented with a hard, enlarging left neck mass. A, Sagittal grey scale US of the left CCA demonstrates an outpouching, consistent with a wide-neck pseudoaneurysm. Note internal echoes (arrow) that fill in approximately half of the pseudoaneurysm lumen, consistent with partial thrombosis. B, A "yin-yang" pattern of swirling blood flow was observed within the residual lumen on color Doppler interrogation. C, Angiography confirmed the presence of a CCA pseudoaneurysm (arrow).

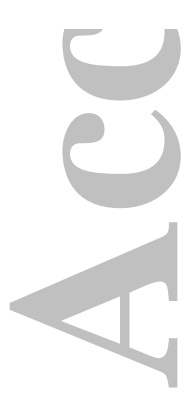

$79 \times 65 \mathrm{~mm}(300 \times 300$ DPI $)$ 


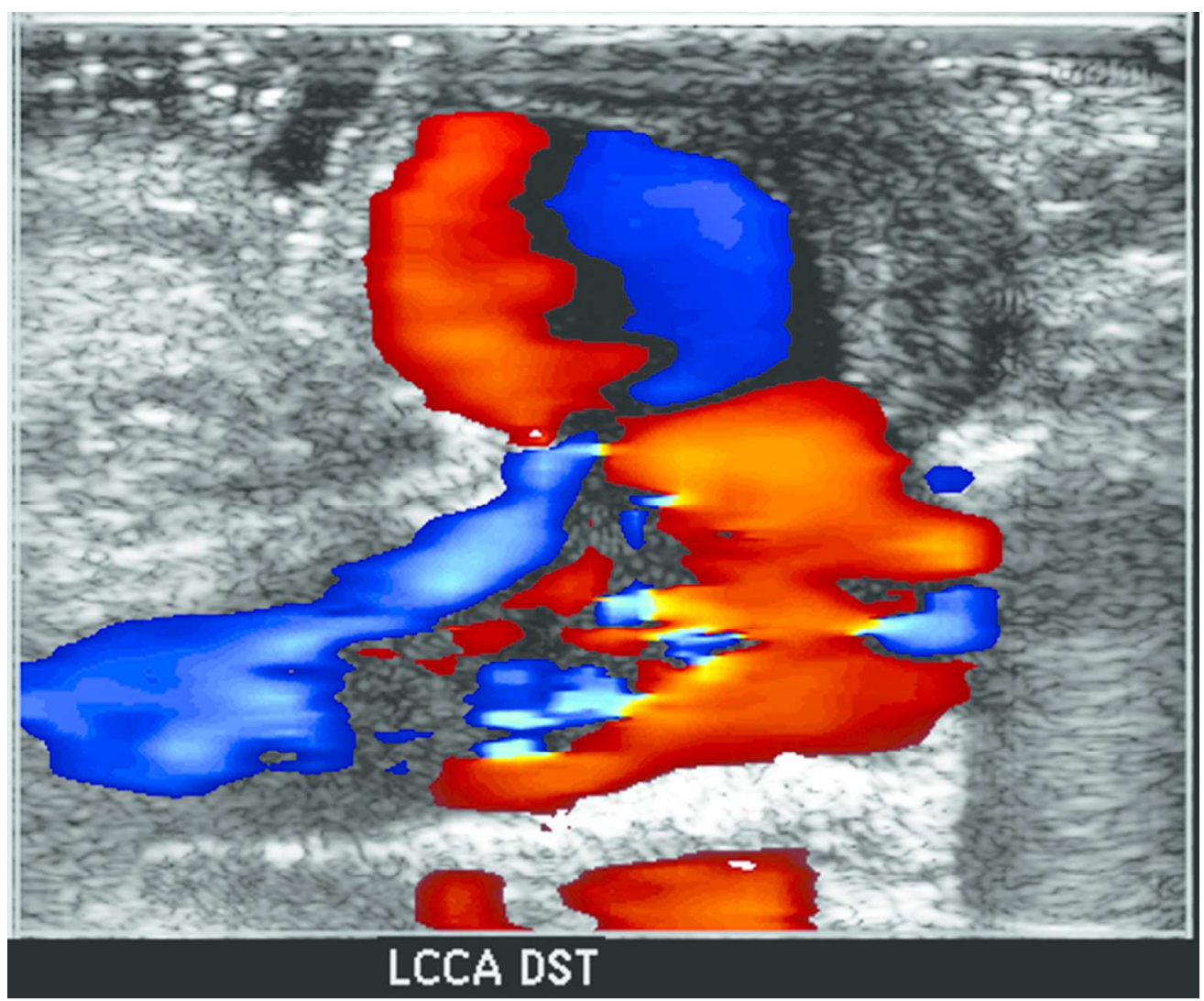

Figure 5B. 83 year old woman status post left CEA three years previously who presented with a hard, enlarging left neck mass. A, Sagittal grey scale US of the left CCA demonstrates an outpouching, consistent with a wide-neck pseudoaneurysm. Note internal echoes (arrow) that fill in approximately half of the pseudoaneurysm lumen, consistent with partial thrombosis. B, A "yin-yang" pattern of swirling blood flow was observed within the residual lumen on color Doppler interrogation. C, Angiography confirmed the presence of a CCA pseudoaneurysm (arrow).

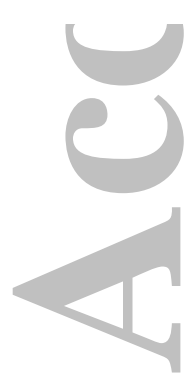

$79 \times 65 \mathrm{~mm}(300 \times 300$ DPI $)$ 


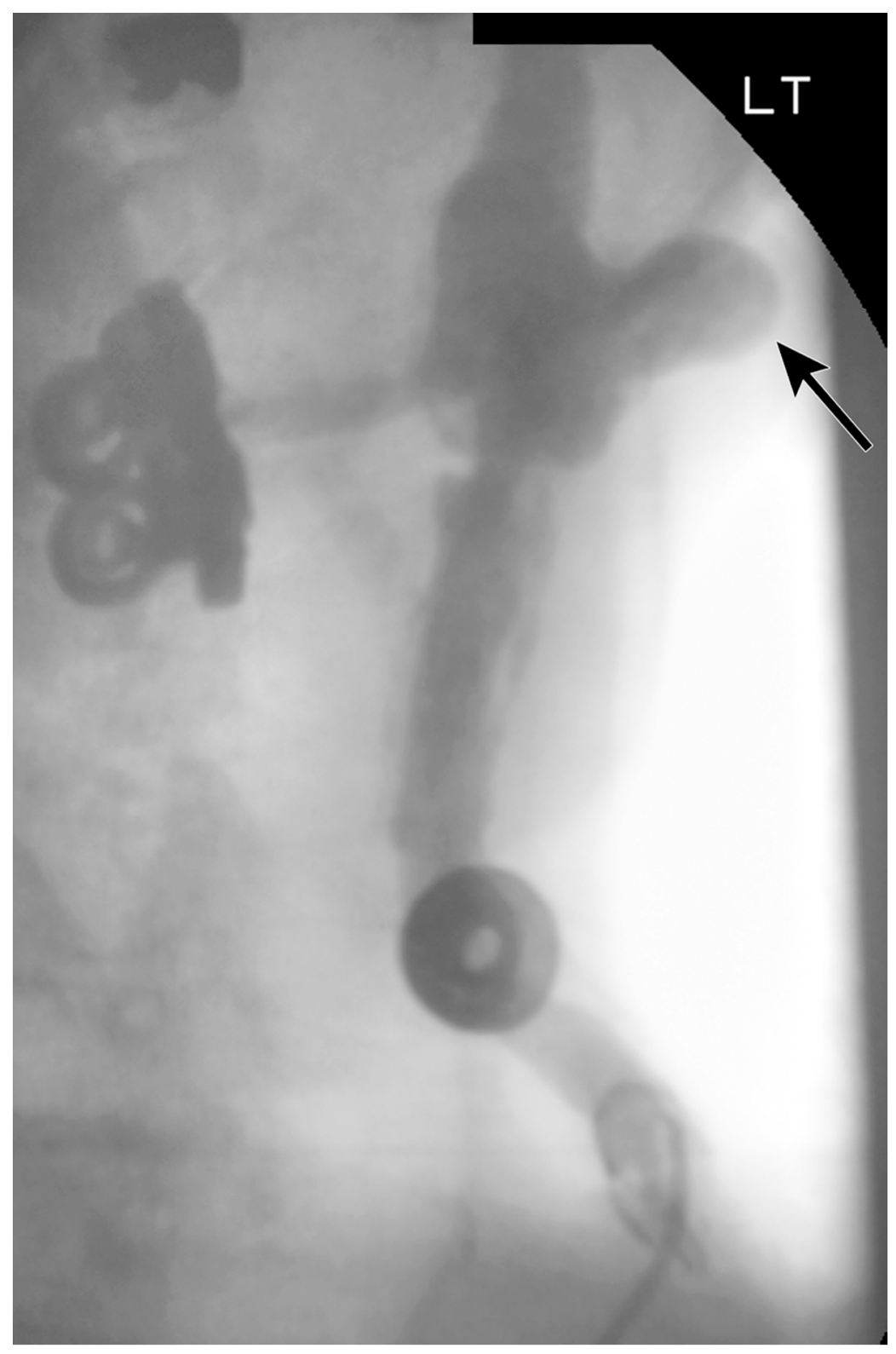

Figure 5C. 83 year old woman status post left CEA three years previously who presented with a hard, enlarging left neck mass. A, Sagittal grey scale US of the left CCA demonstrates an outpouching, consistent with a wide-neck pseudoaneurysm. Note internal echoes (arrow) that fill in approximately half of the pseudoaneurysm lumen, consistent with partial thrombosis. B, A "yin-yang" pattern of swirling blood flow was observed within the residual lumen on color Doppler interrogation. C, Angiography confirmed the presence of a CCA pseudoaneurysm (arrow).

$79 \times 120 \mathrm{~mm}(300 \times 300 \mathrm{DPI})$ 


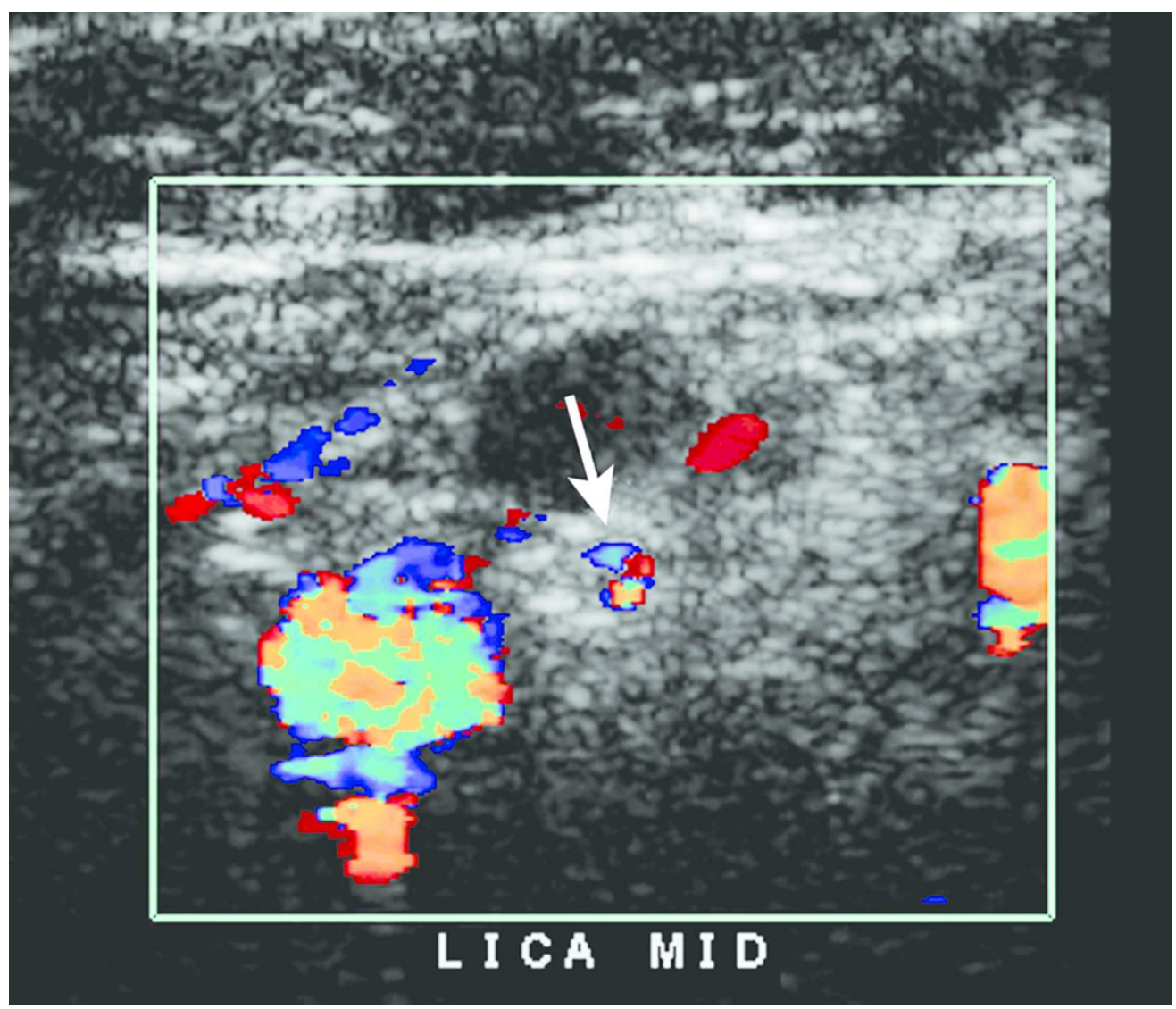

Figure 6A. 60 year old man who experienced a transient ischemic attack a few hours following an uneventful CAS of the left ICA. A and B, Transverse color and sagittal spectral Doppler US of the left ICA show a string sign (arrow in A) and an increased velocity of $273 \mathrm{~cm} / \mathrm{s}$ within the stent. He was subsequently discharged to home on dual antiplatelet therapy. On post procedure day 5, he presented to the emergency room with aphasia. C and D, Repeat sagittal color and spectral Doppler US of the left ICA show no detectable flow

within the stent, concerning for complete thrombosis. E, Axial diffusion weighted MRI of the brain demonstrates multiple foci of increased signal in the left cerebral hemisphere. $F$, These foci are hypointense on the corresponding apparent diffusion coefficient map, consistent with a thromboembolic stroke. G, CTA of the neck performed a few months later for evaluation of the right side redemonstrates the known left ICA stent occlusion (arrow).

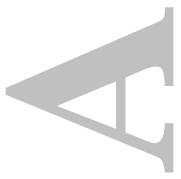

$79 \times 67 \mathrm{~mm}(300 \times 300 \mathrm{DPI})$ 


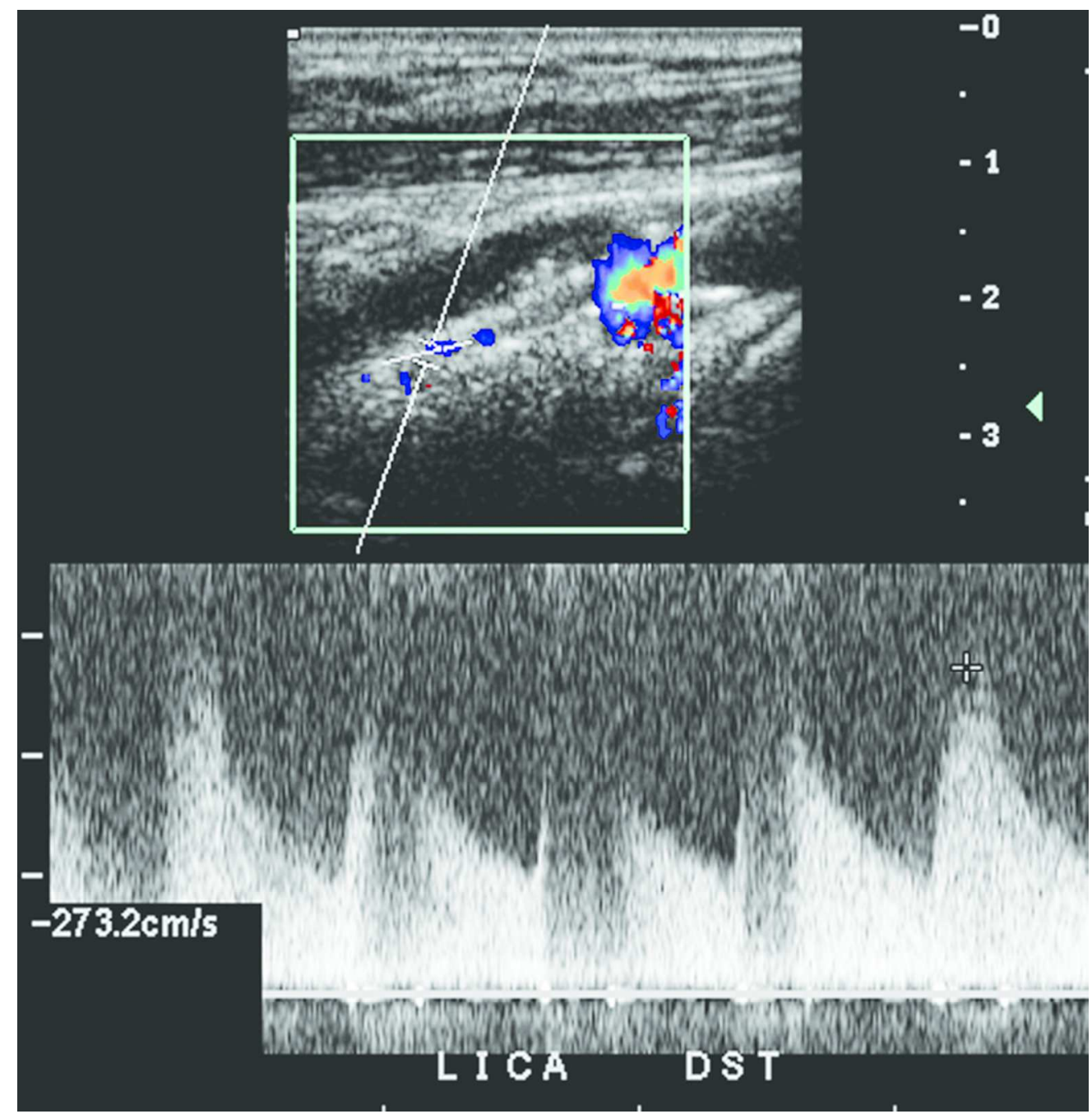

Figure 6B. 60 year old man who experienced a transient ischemic attack a few hours following an uneventful CAS of the left ICA. A and B, Transverse color and sagittal spectral Doppler US of the left ICA show a string sign (arrow in A) and an increased velocity of $273 \mathrm{~cm} / \mathrm{s}$ within the stent. He was subsequently discharged to home on dual antiplatelet therapy. On post procedure day 5, he presented to the emergency room with aphasia. C and D, Repeat sagittal color and spectral Doppler US of the left ICA show no detectable flow within the stent, concerning for complete thrombosis. E, Axial diffusion weighted MRI of the brain demonstrates multiple foci of increased signal in the left cerebral hemisphere. $F$, These foci are hypointense on the corresponding apparent diffusion coefficient map, consistent with a thromboembolic stroke. G, CTA of the neck performed a few months later for evaluation of the right side redemonstrates the known left ICA stent occlusion (arrow).

$79 \times 81 \mathrm{~mm}(300 \times 300$ DPI $)$ 


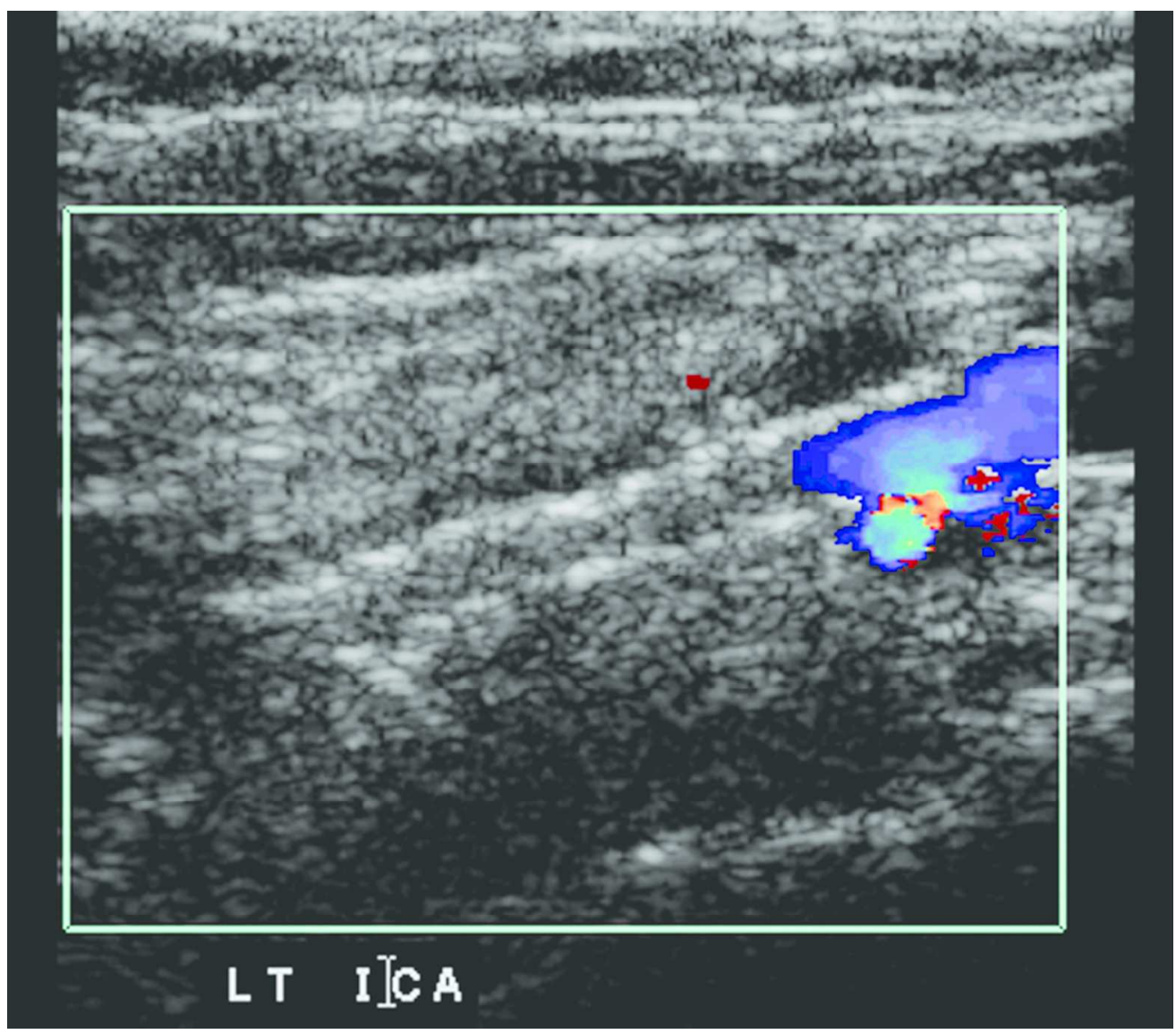

Figure 6C. 60 year old man who experienced a transient ischemic attack a few hours following an uneventful CAS of the left ICA. A and B, Transverse color and sagittal spectral Doppler US of the left ICA show a string sign (arrow in A) and an increased velocity of $273 \mathrm{~cm} / \mathrm{s}$ within the stent. He was subsequently discharged to home on dual antiplatelet therapy. On post procedure day 5, he presented to the emergency

room with aphasia. C and D, Repeat sagittal color and spectral Doppler US of the left ICA show no detectable flow within the stent, concerning for complete thrombosis. $\mathrm{E}$, Axial diffusion weighted MRI of the brain demonstrates multiple foci of increased signal in the left cerebral hemisphere. F, These foci are hypointense on the corresponding apparent diffusion coefficient map, consistent with a thromboembolic stroke. G, CTA of the neck performed a few months later for evaluation of the right side redemonstrates the known left ICA stent occlusion (arrow).

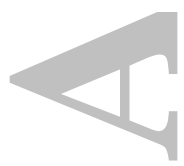

$79 \times 69 \mathrm{~mm}(300 \times 300 \mathrm{DPI})$ 


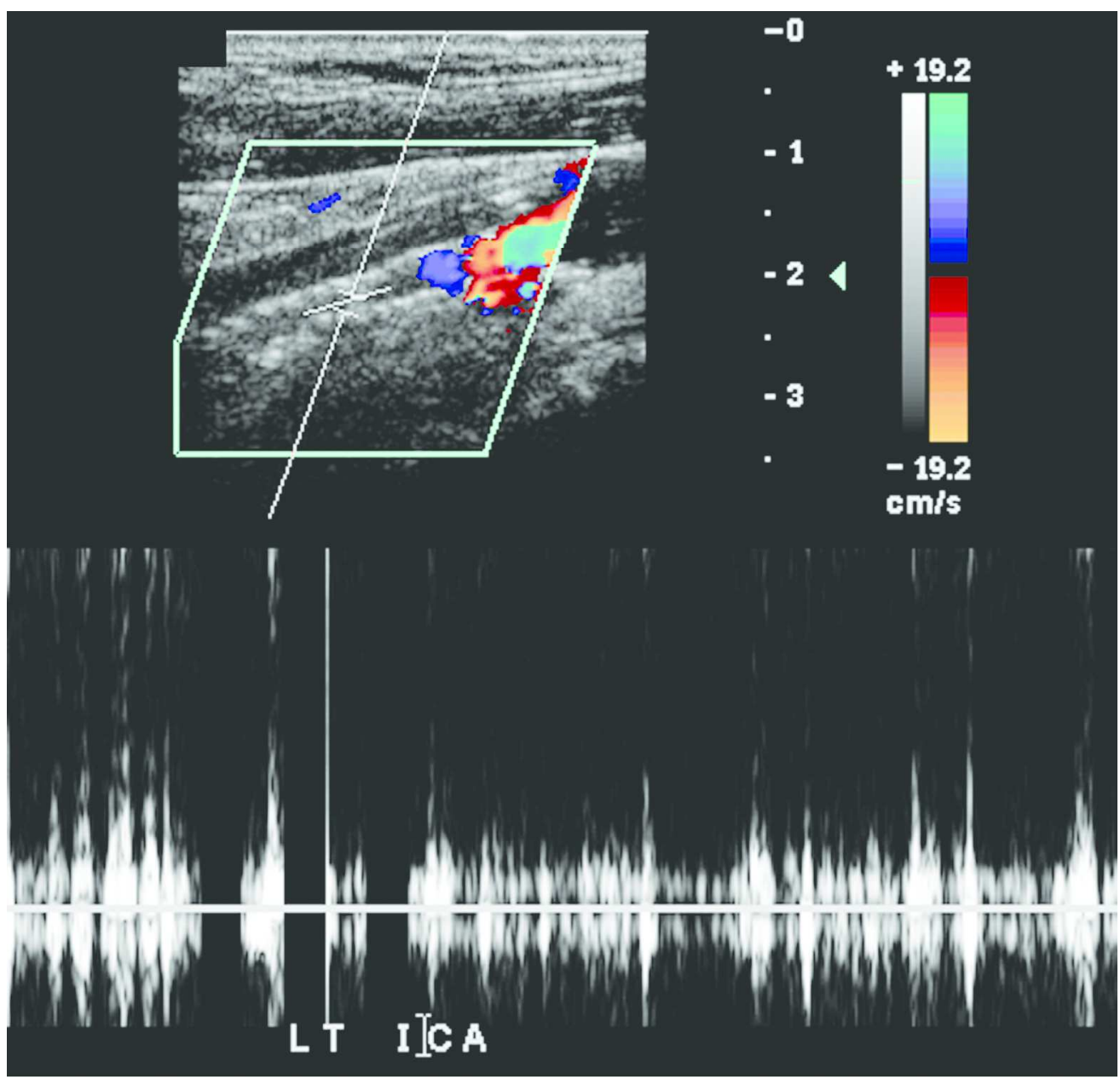

Figure 6D. 60 year old man who experienced a transient ischemic attack a few hours following an uneventful CAS of the left ICA. A and B, Transverse color and sagittal spectral Doppler US of the left ICA show a string sign (arrow in A) and an increased velocity of $273 \mathrm{~cm} / \mathrm{s}$ within the stent. He was subsequently discharged to home on dual antiplatelet therapy. On post procedure day 5, he presented to the emergency

room with aphasia. C and D, Repeat sagittal color and spectral Doppler US of the left ICA show no detectable flow within the stent, concerning for complete thrombosis. E, Axial diffusion weighted MRI of the brain demonstrates multiple foci of increased signal in the left cerebral hemisphere. F, These foci are hypointense on the corresponding apparent diffusion coefficient map, consistent with a thromboembolic stroke. G, CTA of the neck performed a few months later for evaluation of the right side redemonstrates the known left ICA stent occlusion (arrow).

$158 \times 152 \mathrm{~mm}(300 \times 300$ DPI $)$ 


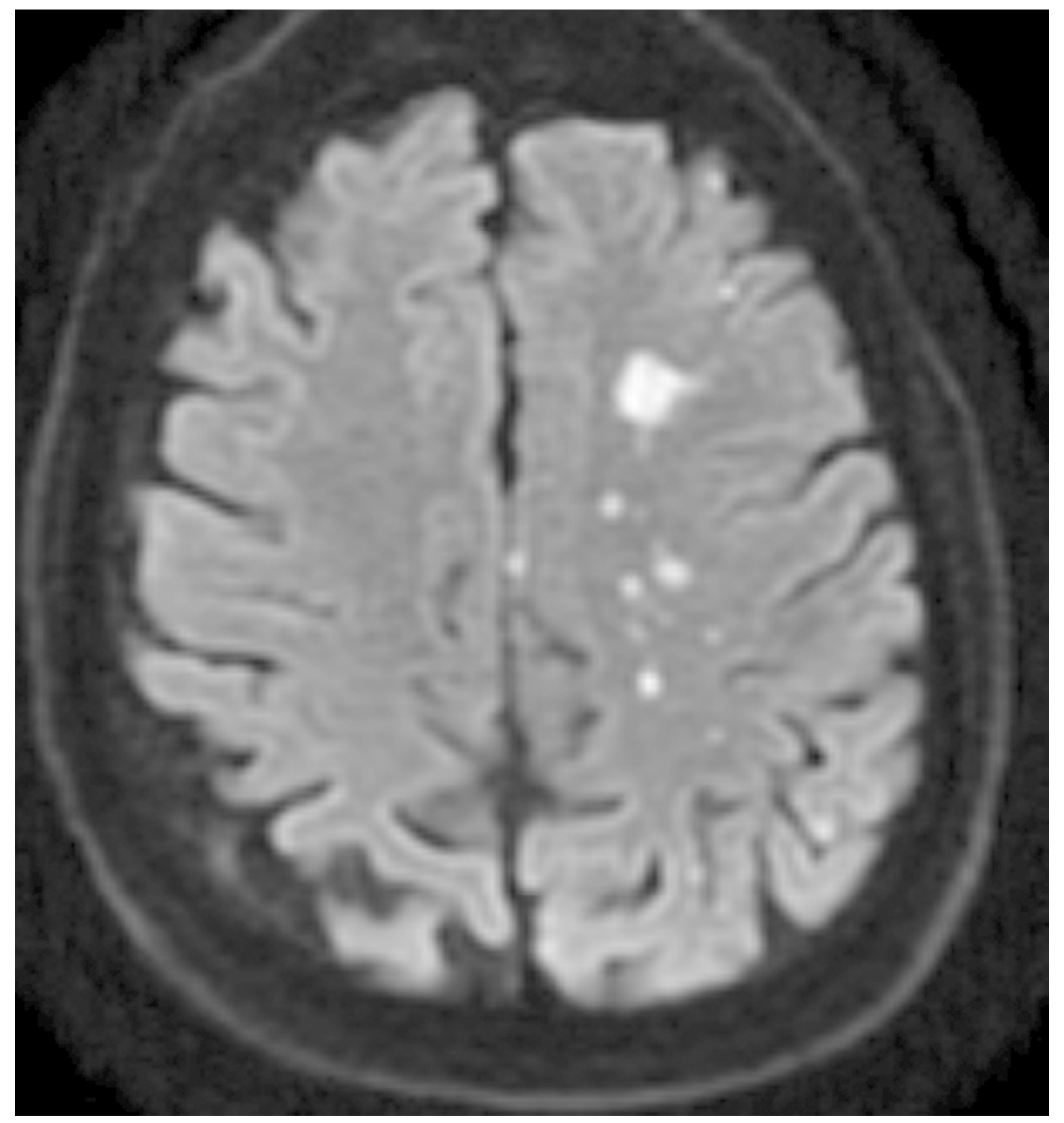

Figure 6E. 60 year old man who experienced a transient ischemic attack a few hours following an uneventful CAS of the left ICA. A and B, Transverse color and sagittal spectral Doppler US of the left ICA show a string sign (arrow in A) and an increased velocity of $273 \mathrm{~cm} / \mathrm{s}$ within the stent. He was subsequently discharged to home on dual antiplatelet therapy. On post procedure day 5, he presented to the emergency room with aphasia. C and D, Repeat sagittal color and spectral Doppler US of the left ICA show no detectable flow within the stent, concerning for complete thrombosis. $\mathrm{E}$, Axial diffusion weighted MRI of the brain demonstrates multiple foci of increased signal in the left cerebral hemisphere. F, These foci are hypointense on the corresponding apparent diffusion coefficient map, consistent with a thromboembolic stroke. G, CTA of the neck performed a few months later for evaluation of the right side redemonstrates the known left ICA stent occlusion (arrow).

$79 \times 85 \mathrm{~mm}(300 \times 300$ DPI $)$ 


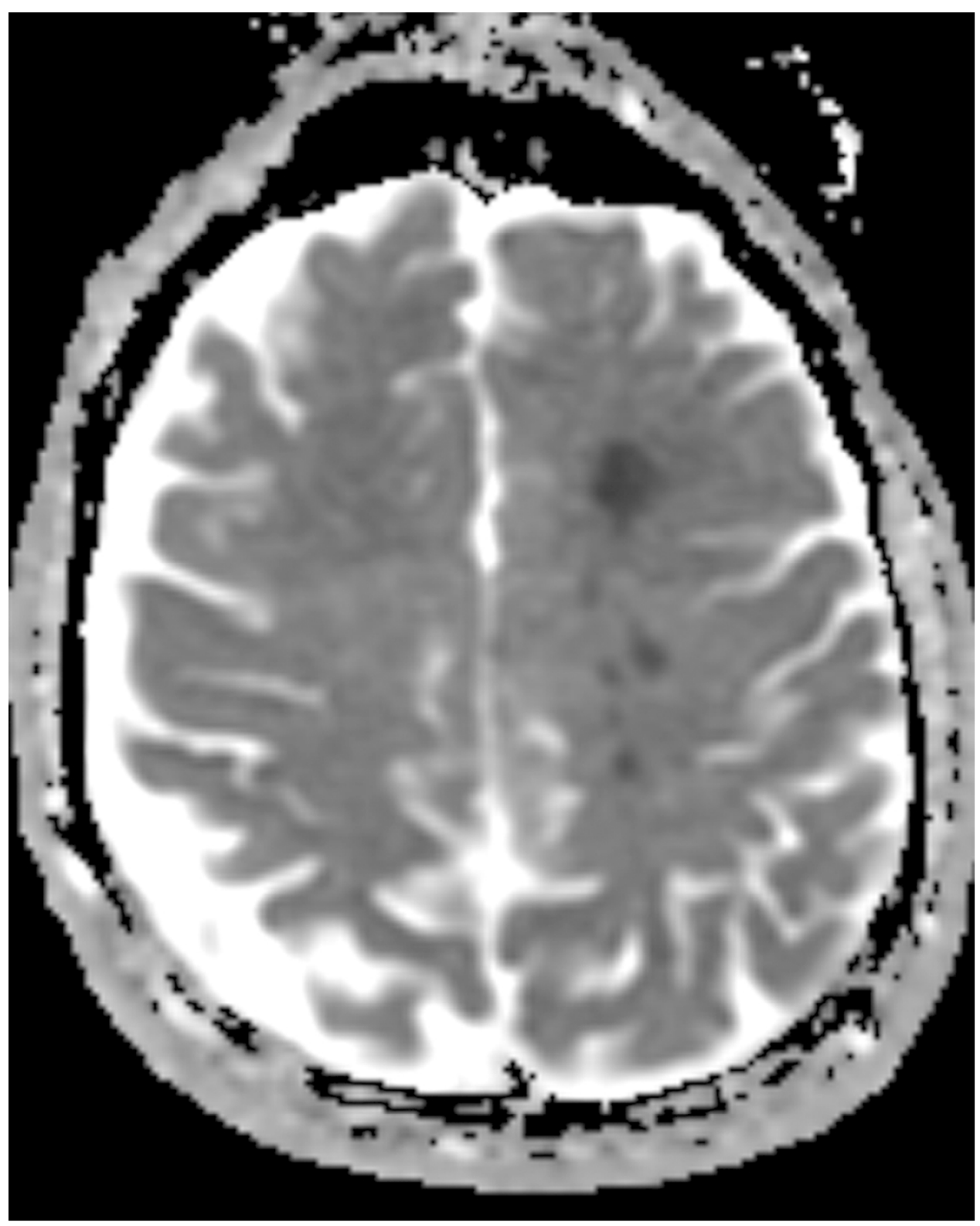

Figure 6F. 60 year old man who experienced a transient ischemic attack a few hours following an uneventful CAS of the left ICA. A and B, Transverse color and sagittal spectral Doppler US of the left ICA show a string sign (arrow in A) and an increased velocity of $273 \mathrm{~cm} / \mathrm{s}$ within the stent. He was subsequently discharged to home on dual antiplatelet therapy. On post procedure day 5, he presented to the emergency

room with aphasia. C and D, Repeat sagittal color and spectral Doppler US of the left ICA show no detectable flow within the stent, concerning for complete thrombosis. E, Axial diffusion weighted MRI of the brain demonstrates multiple foci of increased signal in the left cerebral hemisphere. $F$, These foci are hypointense on the corresponding apparent diffusion coefficient map, consistent with a thromboembolic stroke. G, CTA of the neck performed a few months later for evaluation of the right side redemonstrates the known left ICA stent occlusion (arrow).

$79 \times 99 \mathrm{~mm}(300 \times 300$ DPI $)$ 

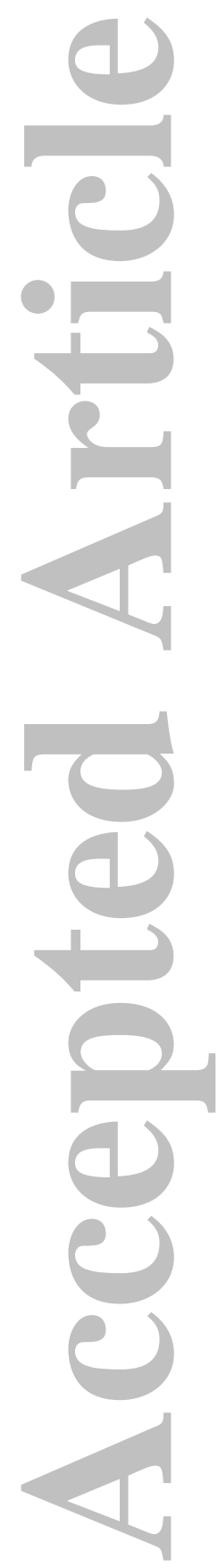

This article is protected by copyright. All rights reserved. 


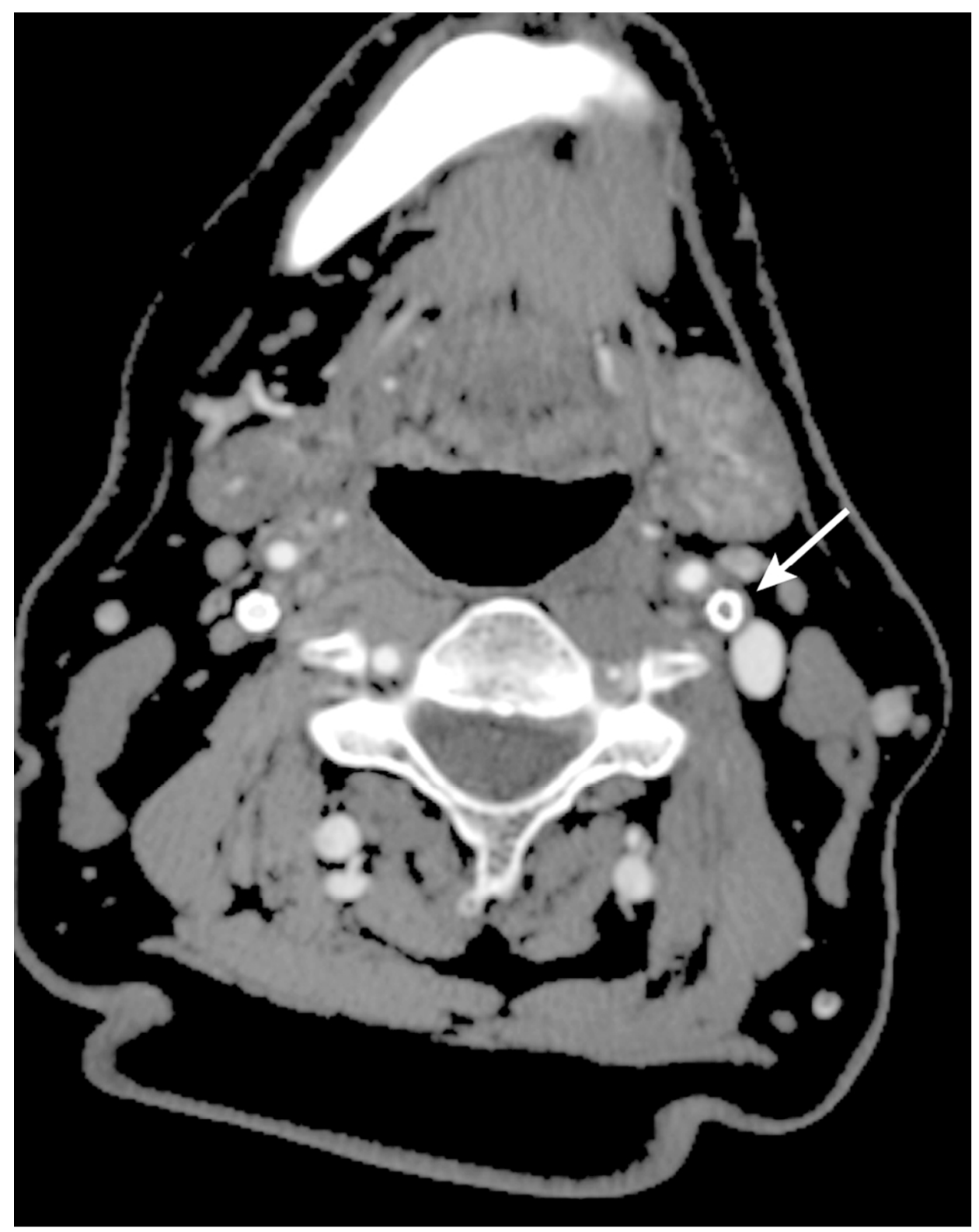

Figure 6G. 60 year old man who experienced a transient ischemic attack a few hours following an uneventful CAS of the left ICA. A and B, Transverse color and sagittal spectral Doppler US of the left ICA show a string sign (arrow in A) and an increased velocity of $273 \mathrm{~cm} / \mathrm{s}$ within the stent. He was subsequently discharged to home on dual antiplatelet therapy. On post procedure day 5, he presented to the emergency

room with aphasia. C and D, Repeat sagittal color and spectral Doppler US of the left ICA show no detectable flow within the stent, concerning for complete thrombosis. E, Axial diffusion weighted MRI of the brain demonstrates multiple foci of increased signal in the left cerebral hemisphere. $F$, These foci are hypointense on the corresponding apparent diffusion coefficient map, consistent with a thromboembolic stroke. G, CTA of the neck performed a few months later for evaluation of the right side redemonstrates the known left ICA stent occlusion (arrow).

$79 \times 100 \mathrm{~mm}(300 \times 300$ DPI $)$ 

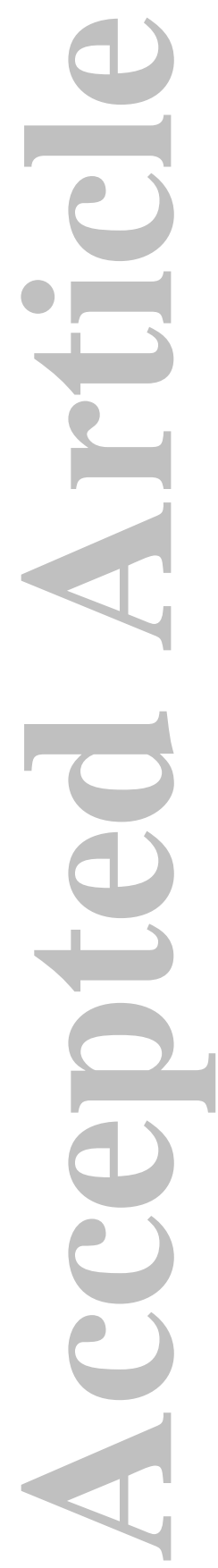

This article is protected by copyright. All rights reserved. 


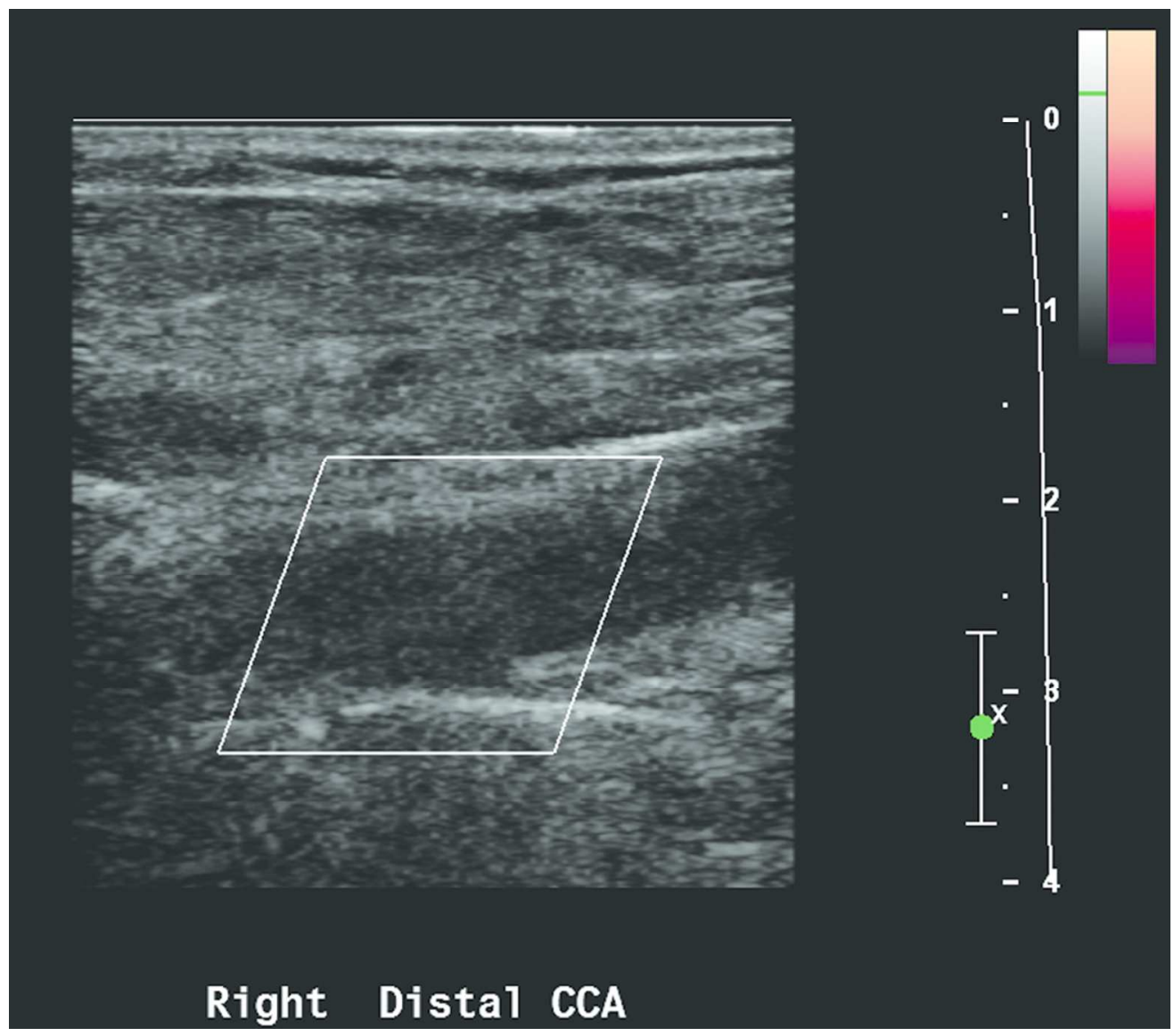

Figure 7A. 82 year old woman with prior surgical history of left CEA, who developed left sided weakness, left facial droop, and dysarthria 4 hours following right CEA. A There is no demonstrable flow within the right distal CCA on sagittal color Doppler US, suggestive of complete thrombosis. B, Axial diffusion weighted MRI of the brain demonstrates increased signal in portions of the right parietal and frontal lobes, consistent with an acute infarction in the territory of the right middle cerebral artery.

$79 \times 69 \mathrm{~mm}(300 \times 300 \mathrm{DPI})$

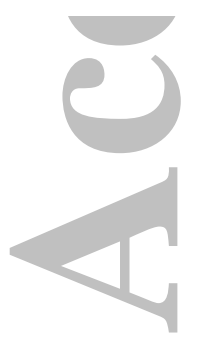




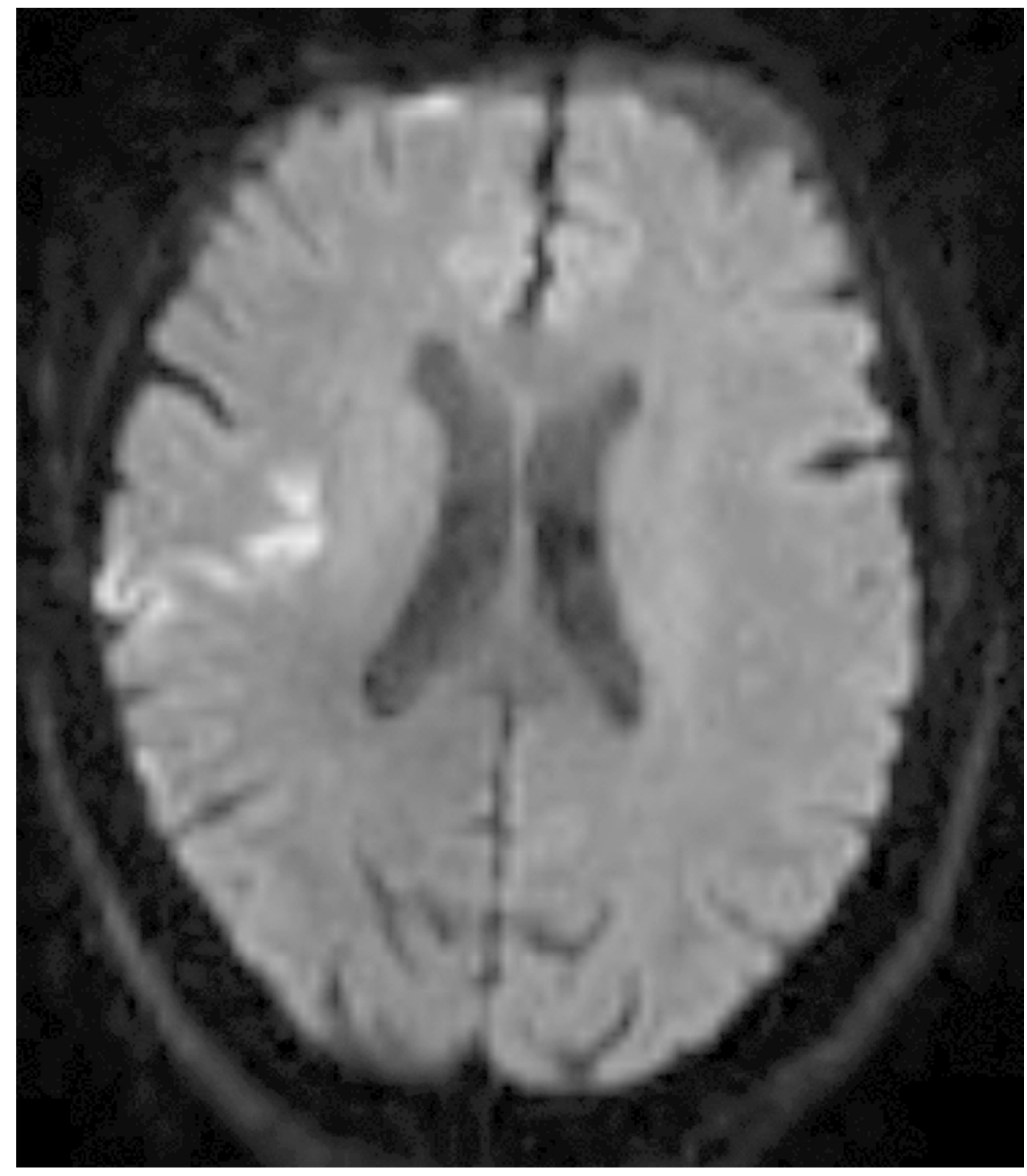

Figure 7B. 82 year old woman with prior surgical history of left CEA, who developed left sided weakness, left facial droop, and dysarthria 4 hours following right CEA. A There is no demonstrable flow within the right distal CCA on sagittal color Doppler US, suggestive of complete thrombosis. B, Axial diffusion weighted MRI of the brain demonstrates increased signal in portions of the right parietal and frontal lobes, consistent with an acute infarction in the territory of the right middle cerebral artery.

$79 \times 91 \mathrm{~mm}(300 \times 300$ DPI $)$ 


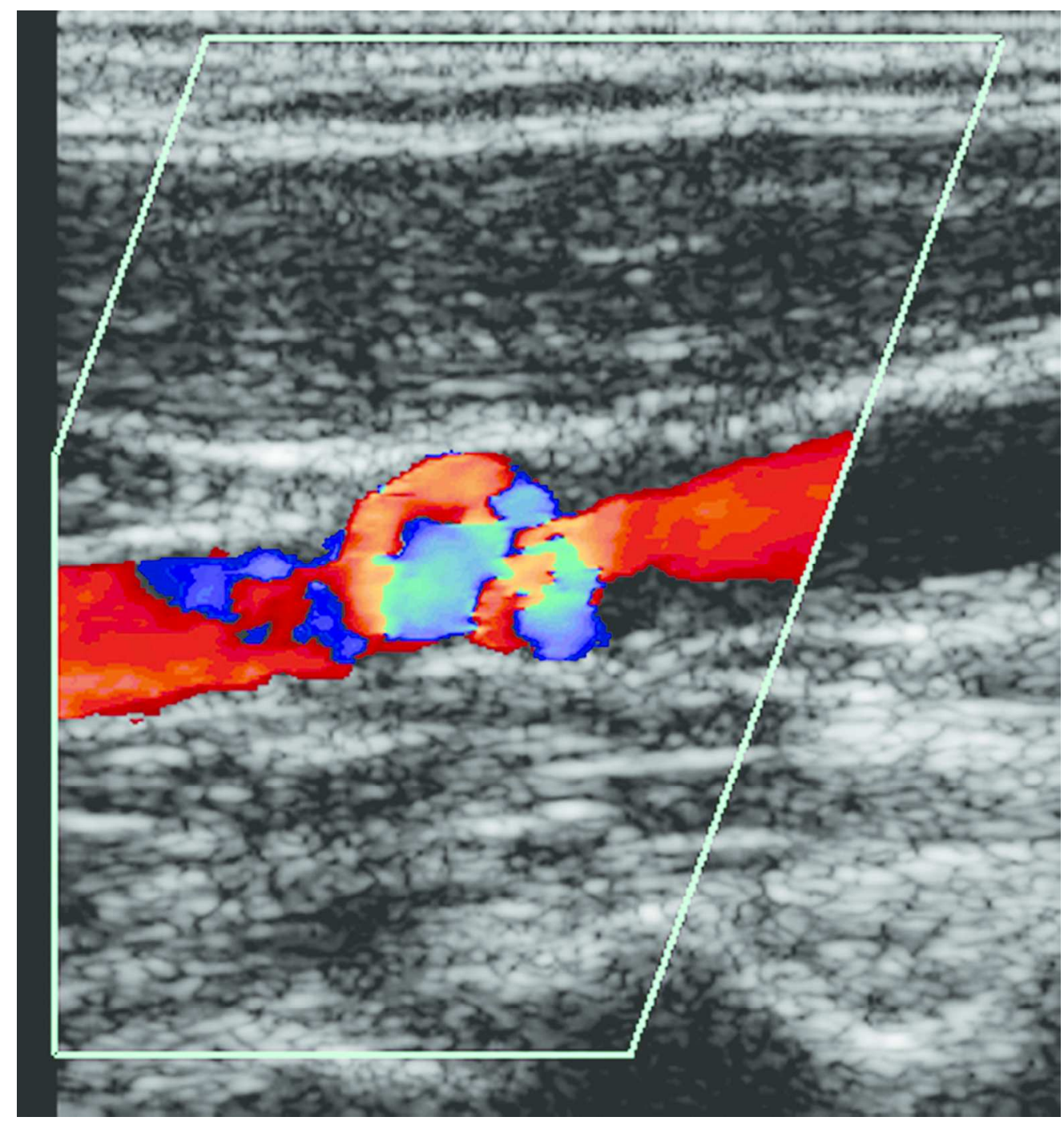

Figure 8A. 65 year old man who is status post left CEA 9 years previously. A and B, Sagittal color and spectral Doppler US demonstrate color aliasing with an increased flow velocity of $234 \mathrm{~cm} / \mathrm{s}$ within the left proximal ICA, concerning for a high grade stenosis. C, DSA confirms a high-grade focal left proximal ICA stenosis (arrow). The patient subsequently underwent a technically successful CAS (not shown). D and E, Sagittal color and spectral Doppler US 1 month later demonstrate a widely patent stent with a normal peak systolic velocity of $106 \mathrm{~cm} / \mathrm{s}$. F, Repeat angiogram 2 years later shows high-grade focal in-stent restenosis (arrow). The patient was treated with angioplasty (not shown).

$79 \times 84 \mathrm{~mm}(300 \times 300$ DPI $)$ 


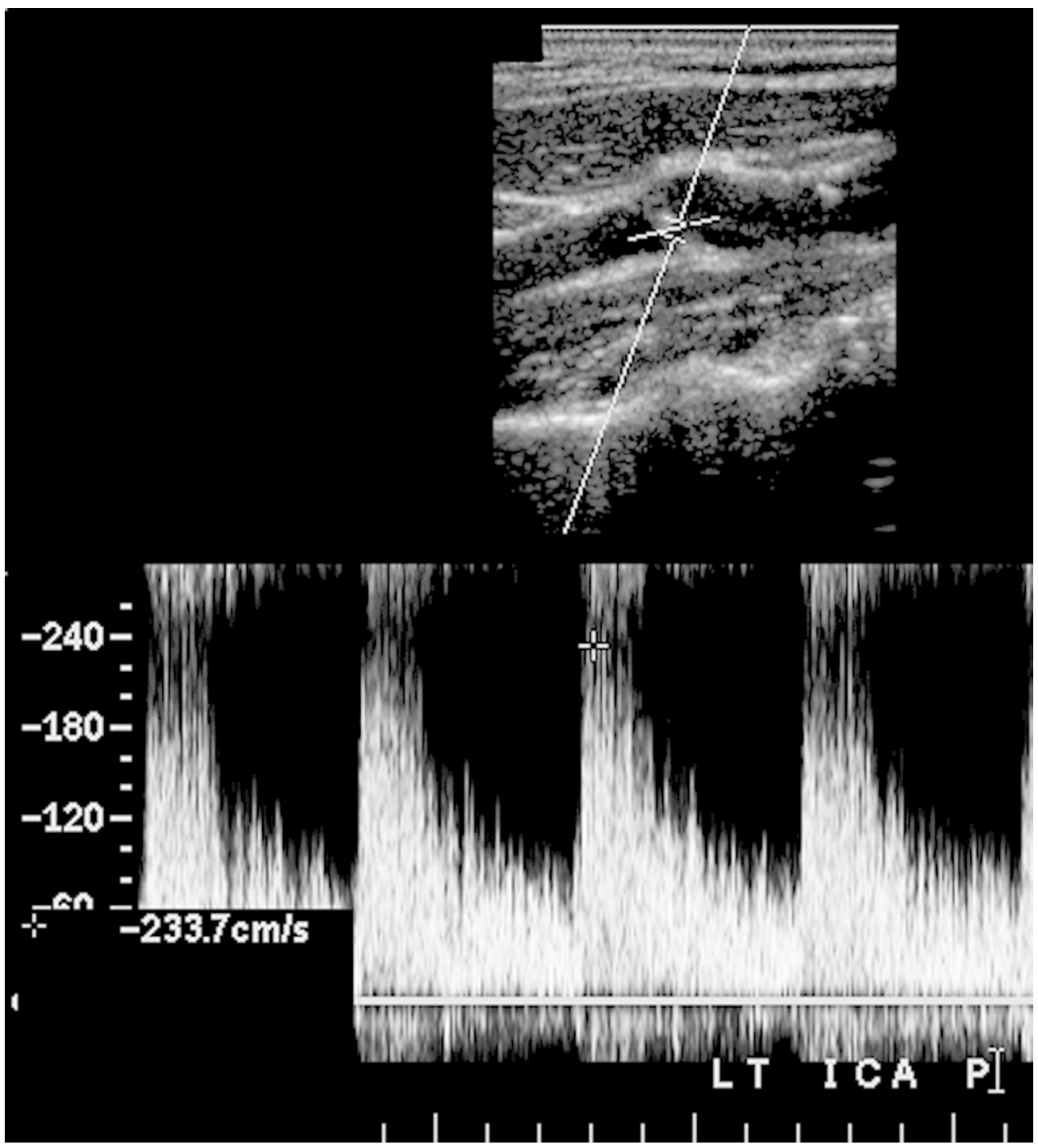

Figure 8B. 65 year old man who is status post left CEA 9 years previously. A and B, Sagittal color and spectral Doppler US demonstrate color aliasing with an increased flow velocity of $234 \mathrm{~cm} / \mathrm{s}$ within the left proximal ICA, concerning for a high grade stenosis. C, DSA confirms a high-grade focal left proximal ICA stenosis (arrow). The patient subsequently underwent a technically successful CAS (not shown). D and E, Sagittal color and spectral Doppler US 1 month later demonstrate a widely patent stent with a normal peak systolic velocity of $106 \mathrm{~cm} / \mathrm{s}$. F, Repeat angiogram 2 years later shows high-grade focal in-stent restenosis (arrow). The patient was treated with angioplasty (not shown).

$79 \times 87 \mathrm{~mm}(300 \times 300$ DPI $)$ 


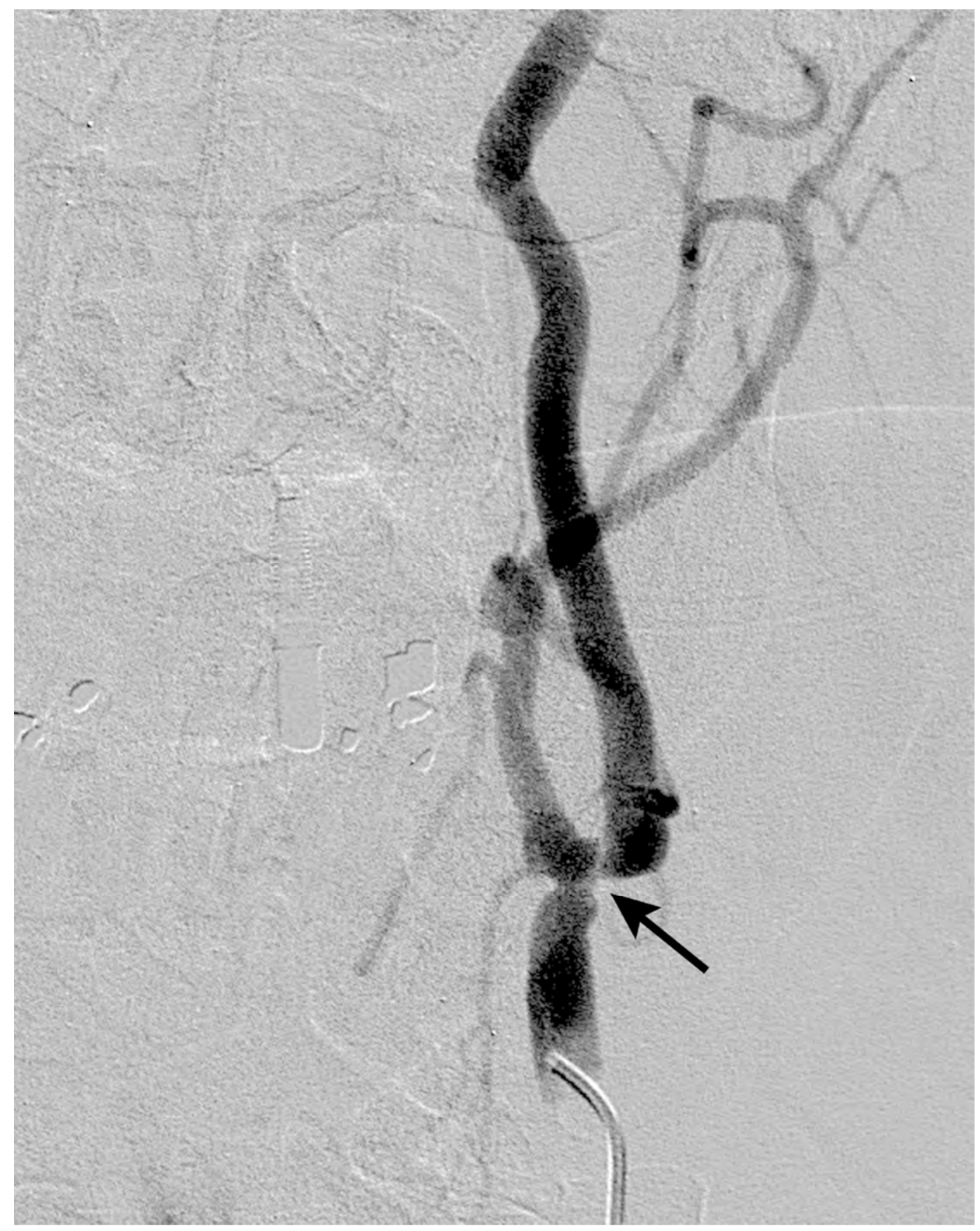

Figure 8C. 65 year old man who is status post left CEA 9 years previously. A and B, Sagittal color and spectral Doppler US demonstrate color aliasing with an increased flow velocity of $234 \mathrm{~cm} / \mathrm{s}$ within the left proximal ICA, concerning for a high grade stenosis. C, DSA confirms a high-grade focal left proximal ICA stenosis (arrow). The patient subsequently underwent a technically successful CAS (not shown). D and E, Sagittal color and spectral Doppler US 1 month later demonstrate a widely patent stent with a normal peak systolic velocity of $106 \mathrm{~cm} / \mathrm{s}$. F, Repeat angiogram 2 years later shows high-grade focal in-stent restenosis (arrow). The patient was treated with angioplasty (not shown).

$79 \times 100 \mathrm{~mm}(300 \times 300 \mathrm{DPI})$ 


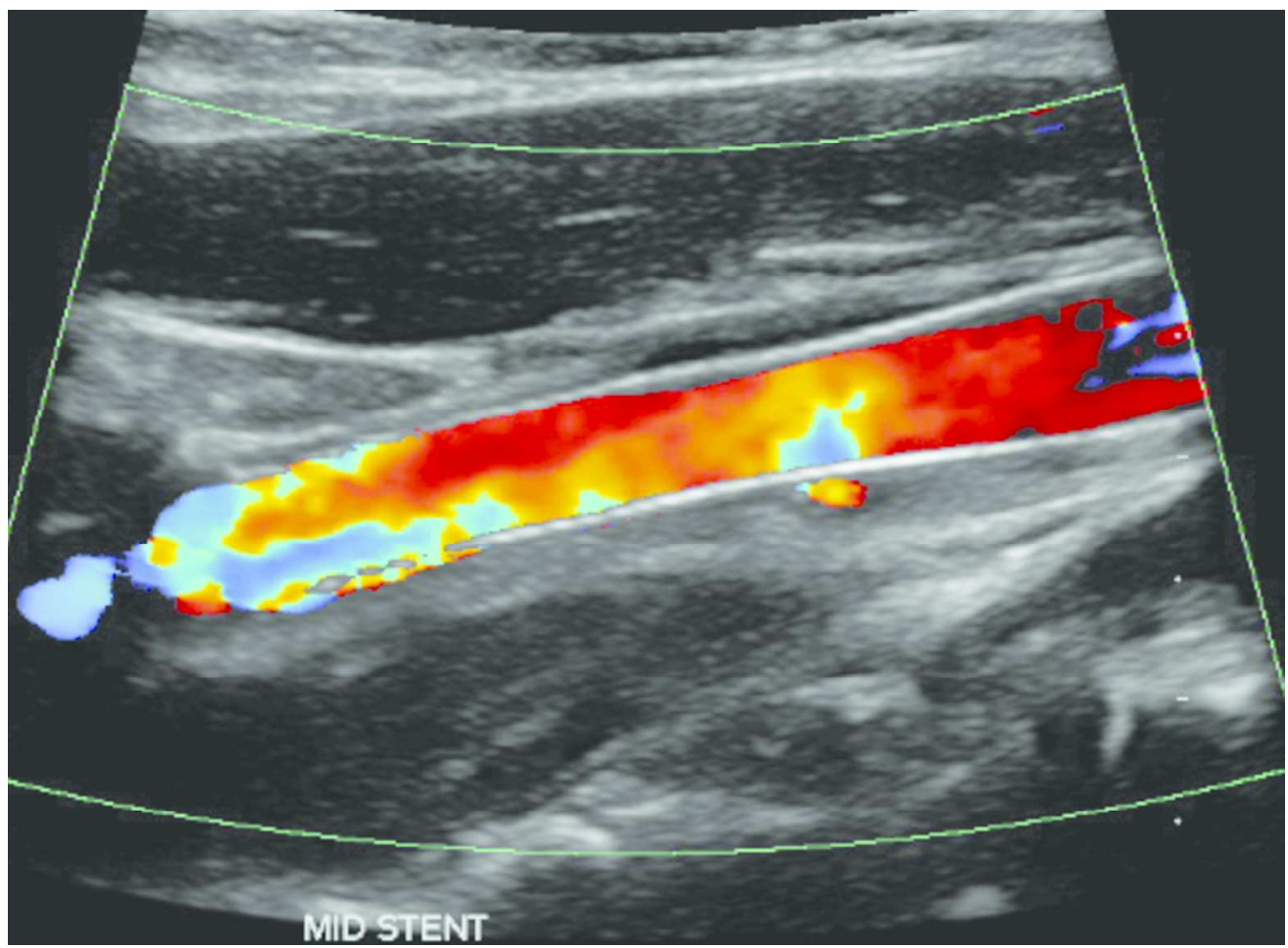

Figure 8D. 65 year old man who is status post left CEA 9 years previously. A and B, Sagittal color and spectral Doppler US demonstrate color aliasing with an increased flow velocity of $234 \mathrm{~cm} / \mathrm{s}$ within the left proximal ICA, concerning for a high grade stenosis. C, DSA confirms a high-grade focal left proximal ICA stenosis (arrow). The patient subsequently underwent a technically successful CAS (not shown). D and $E_{\text {, }}$ Sagittal color and spectral Doppler US 1 month later demonstrate a widely patent stent with a normal peak systolic velocity of $106 \mathrm{~cm} / \mathrm{s}$. F, Repeat angiogram 2 years later shows high-grade focal in-stent restenosis (arrow). The patient was treated with angioplasty (not shown).

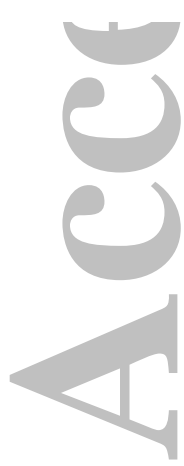

$79 \times 58 \mathrm{~mm}(300 \times 300$ DPI $)$ 


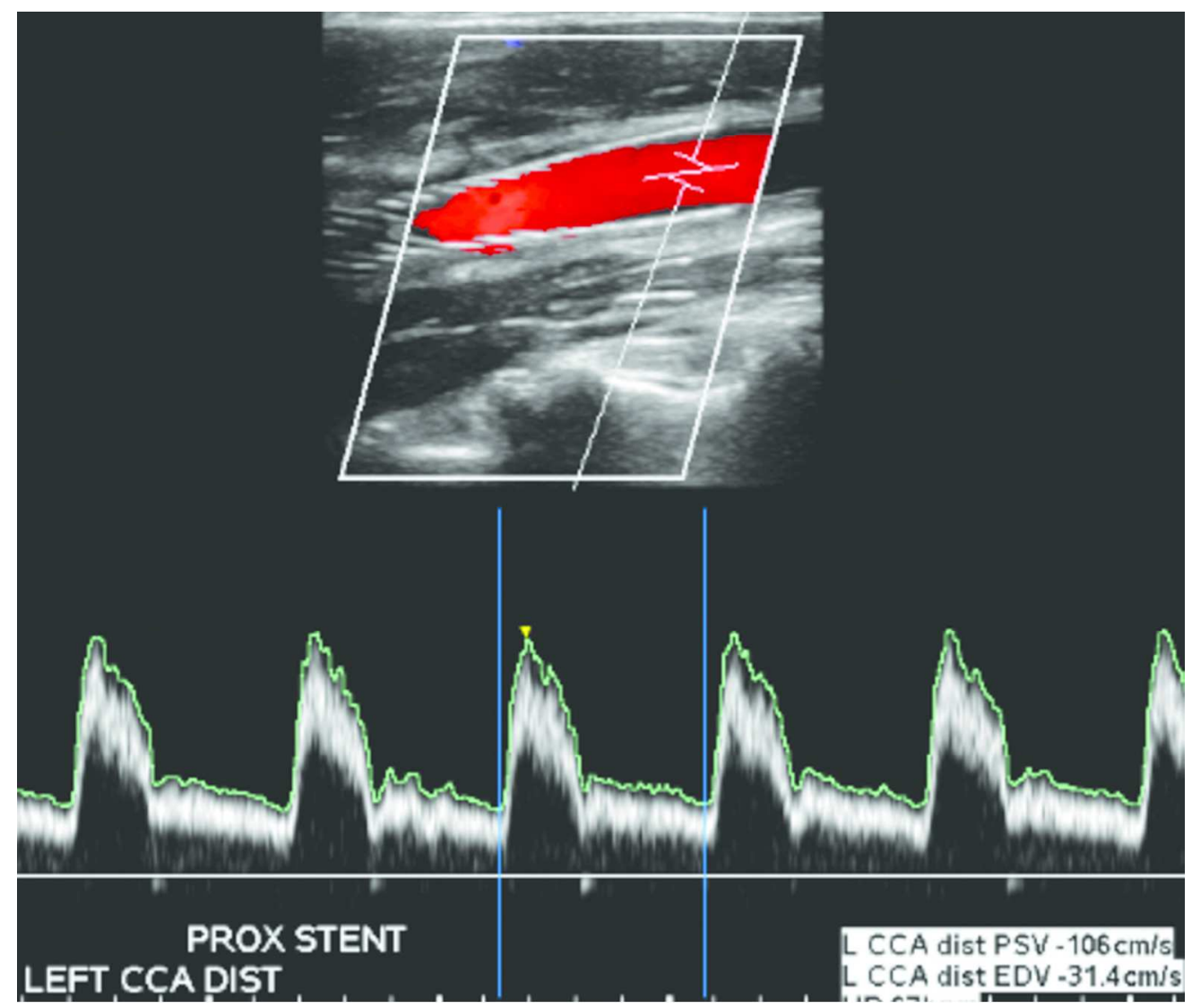

Figure 8E. 65 year old man who is status post left CEA 9 years previously. A and B, Sagittal color and spectral Doppler US demonstrate color aliasing with an increased flow velocity of $234 \mathrm{~cm} / \mathrm{s}$ within the left proximal ICA, concerning for a high grade stenosis. C, DSA confirms a high-grade focal left proximal ICA stenosis (arrow). The patient subsequently underwent a technically successful CAS (not shown). D and E, Sagittal color and spectral Doppler US 1 month later demonstrate a widely patent stent with a normal peak systolic velocity of $106 \mathrm{~cm} / \mathrm{s}$. F, Repeat angiogram 2 years later shows high-grade focal in-stent restenosis (arrow). The patient was treated with angioplasty (not shown).

$79 \times 67 \mathrm{~mm}(300 \times 300 \mathrm{DPI})$

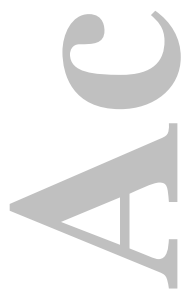




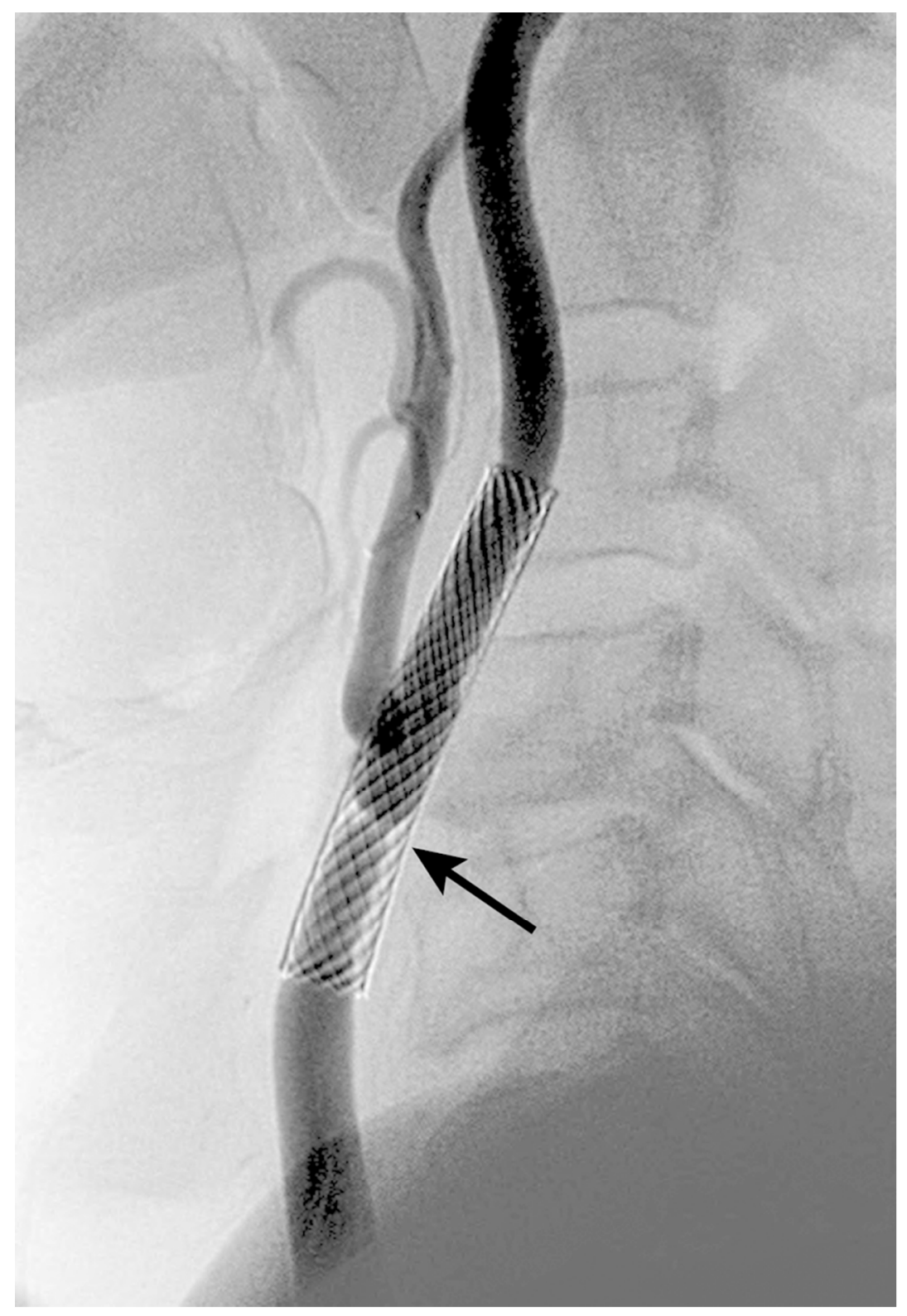

Figure 8F. 65 year old man who is status post left CEA 9 years previously. A and B, Sagittal color and spectral Doppler US demonstrate color aliasing with an increased flow velocity of $234 \mathrm{~cm} / \mathrm{s}$ within the left proximal ICA, concerning for a high grade stenosis. C, DSA confirms a high-grade focal left proximal ICA stenosis (arrow). The patient subsequently underwent a technically successful CAS (not shown). D and E, Sagittal color and spectral Doppler US 1 month later demonstrate a widely patent stent with a normal peak systolic velocity of $106 \mathrm{~cm} / \mathrm{s}$. F, Repeat angiogram 2 years later shows high-grade focal in-stent restenosis (arrow). The patient was treated with angioplasty (not shown).

$79 \times 116 \mathrm{~mm}(300 \times 300 \mathrm{DPI})$ 


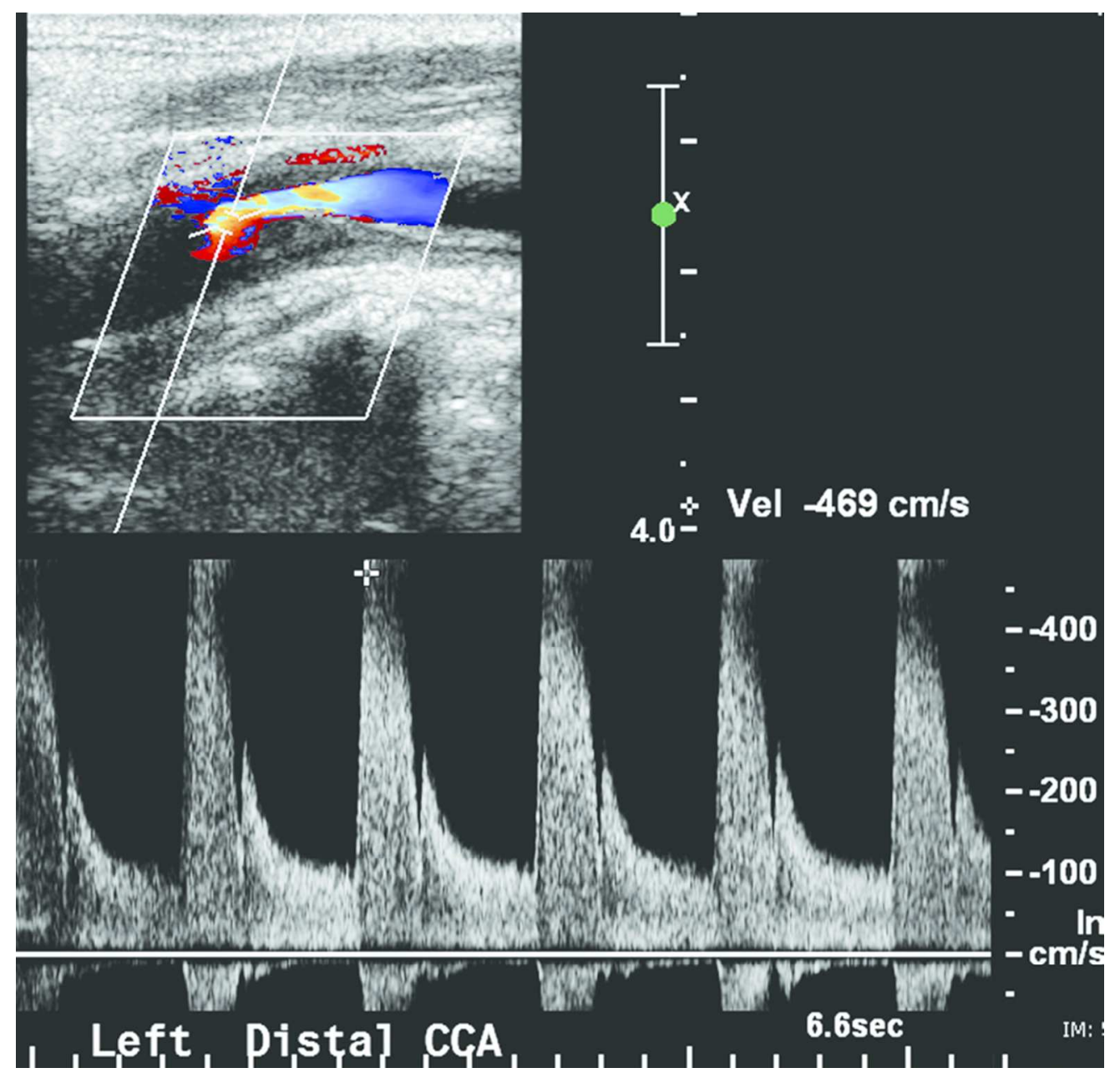

Figure 9A. 73 year old man with history of prior strokes, and who had also received radiation therapy for throat cancer. A, Sagittal spectral Doppler US demonstrates a high-grade stenosis within the left distal CCA with a peak systolic velocity of $469 \mathrm{~cm} / \mathrm{s}$. B, Severe CCA stenosis is confirmed on 3D reconstructed CTA (arrow). C, The stenosis is also demonstrated on DSA (arrow). The patient underwent successful CAS with angioplasty. $D$ and $E$, Post-stent angiogram demonstrates a focal bulge in the location of the prior stenosis

(arrow), consistent with stent deformation without evidence of a fracture. A few days following the procedure, the patient had a transient ischemic attack. F, Grey scale US demonstrates the mildy deformed but intact stent struts. G, Sagittal color and spectral Doppler US of the proximal left ICA demonstrates interval appearance of a hypoechoic area at the origin of the left ICA (arrow) with an elevated peak systolic velocity of $191 \mathrm{~cm} / \mathrm{s}$ (scale not shown), consistent with an acute thrombus. H, Diffusion weighted MRI of the brain shows multiple hyperintense foci confined to the left cerebral hemisphere, consistent with multiple thromboemboli from a carotid source.

$79 \times 77 m m(300 \times 300$ DPI) 


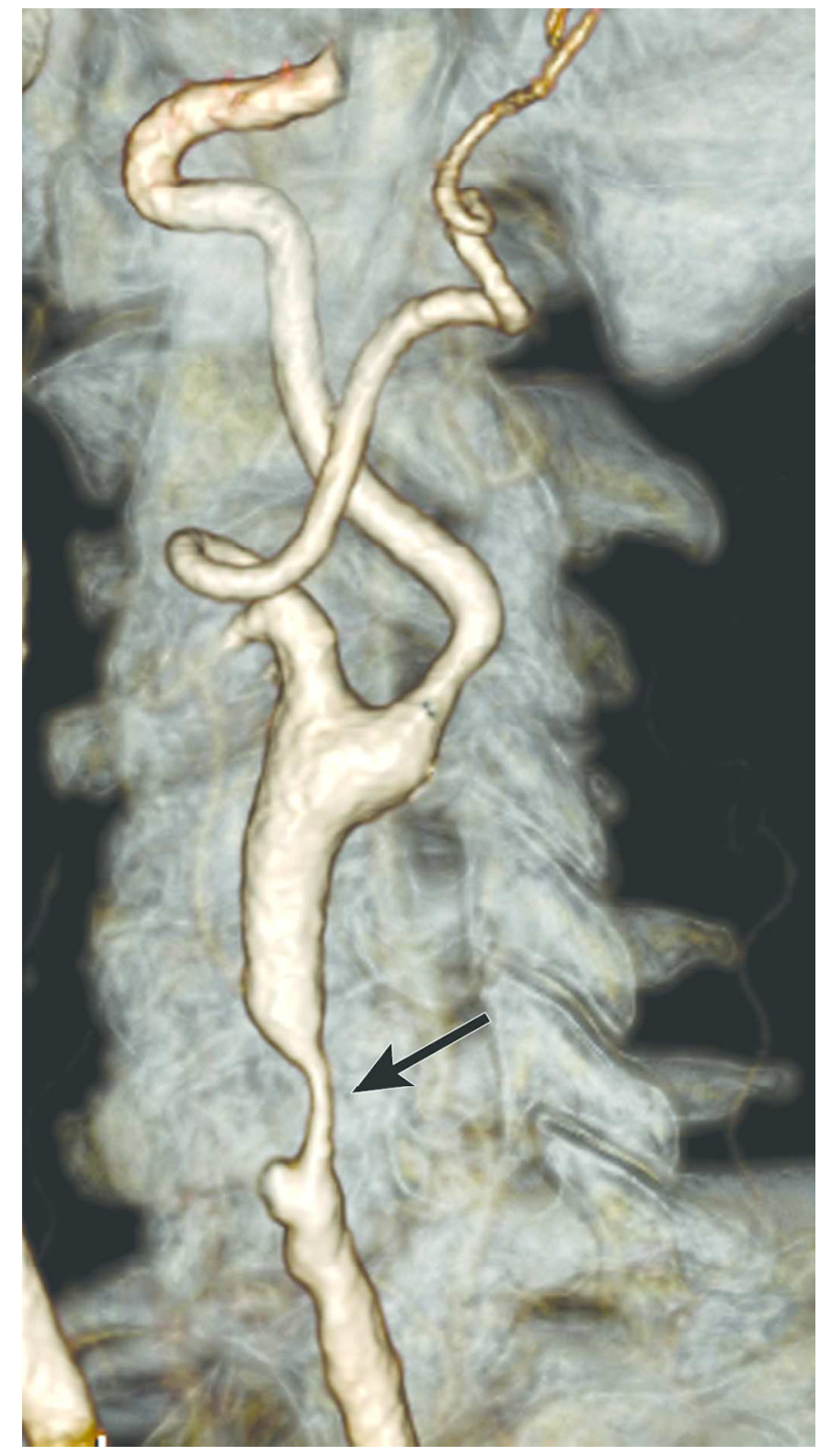

Figure 9B. 73 year old man with history of prior strokes, and who had also received radiation therapy for throat cancer. A, Sagittal spectral Doppler US demonstrates a high-grade stenosis within the left distal CCA with a peak systolic velocity of $469 \mathrm{~cm} / \mathrm{s}$. B, Severe CCA stenosis is confirmed on 3D reconstructed CTA (arrow). C, The stenosis is also demonstrated on DSA (arrow). The patient underwent successful CAS with angioplasty. D and $E$, Post-stent angiogram demonstrates a focal bulge in the location of the prior stenosis

(arrow), consistent with stent deformation without evidence of a fracture. A few days following the procedure, the patient had a transient ischemic attack. F, Grey scale US demonstrates the mildy deformed but intact stent struts. G, Sagittal color and spectral Doppler US of the proximal left ICA demonstrates interval appearance of a hypoechoic area at the origin of the left ICA (arrow) with an elevated peak systolic velocity of $191 \mathrm{~cm} / \mathrm{s}$ (scale not shown), consistent with an acute thrombus. H, Diffusion weighted MRI of the brain shows multiple hyperintense foci confined to the left cerebral hemisphere, consistent with multiple thromboemboli from a carotid source. 
$79 \times 143 \mathrm{~mm}(300 \times 300$ DPI $)$
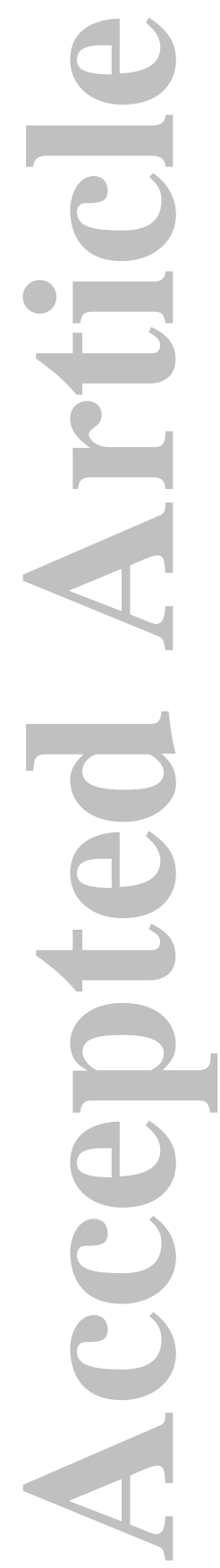

This article is protected by copyright. All rights reserved. 


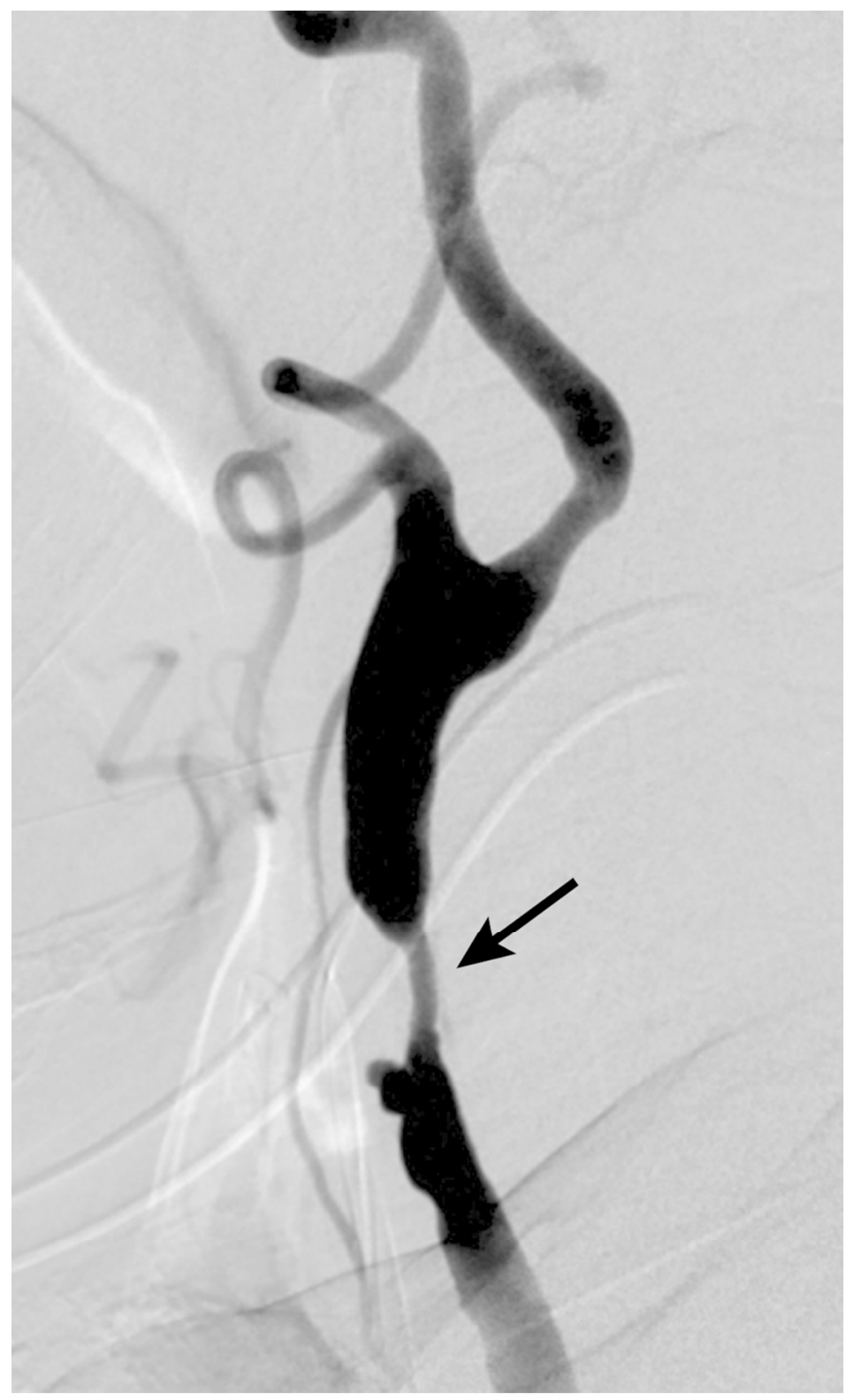

Figure 9C. 73 year old man with history of prior strokes, and who had also received radiation therapy for throat cancer. A, Sagittal spectral Doppler US demonstrates a high-grade stenosis within the left distal CCA with a peak systolic velocity of $469 \mathrm{~cm} / \mathrm{s}$. B, Severe CCA stenosis is confirmed on 3D reconstructed CTA (arrow). C, The stenosis is also demonstrated on DSA (arrow). The patient underwent successful CAS with angioplasty. D and $E$, Post-stent angiogram demonstrates a focal bulge in the location of the prior stenosis

(arrow), consistent with stent deformation without evidence of a fracture. A few days following the procedure, the patient had a transient ischemic attack. F, Grey scale US demonstrates the mildy deformed but intact stent struts. G, Sagittal color and spectral Doppler US of the proximal left ICA demonstrates interval appearance of a hypoechoic area at the origin of the left ICA (arrow) with an elevated peak systolic velocity of $191 \mathrm{~cm} / \mathrm{s}$ (scale not shown), consistent with an acute thrombus. H, Diffusion weighted MRI of the brain shows multiple hyperintense foci confined to the left cerebral hemisphere, consistent with multiple thromboemboli from a carotid source. 
$79 \times 131 \mathrm{~mm}(300 \times 300$ DPI $)$
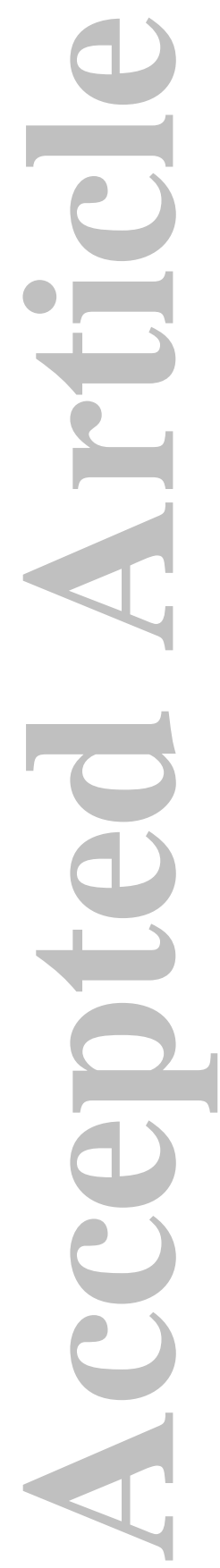

This article is protected by copyright. All rights reserved. 


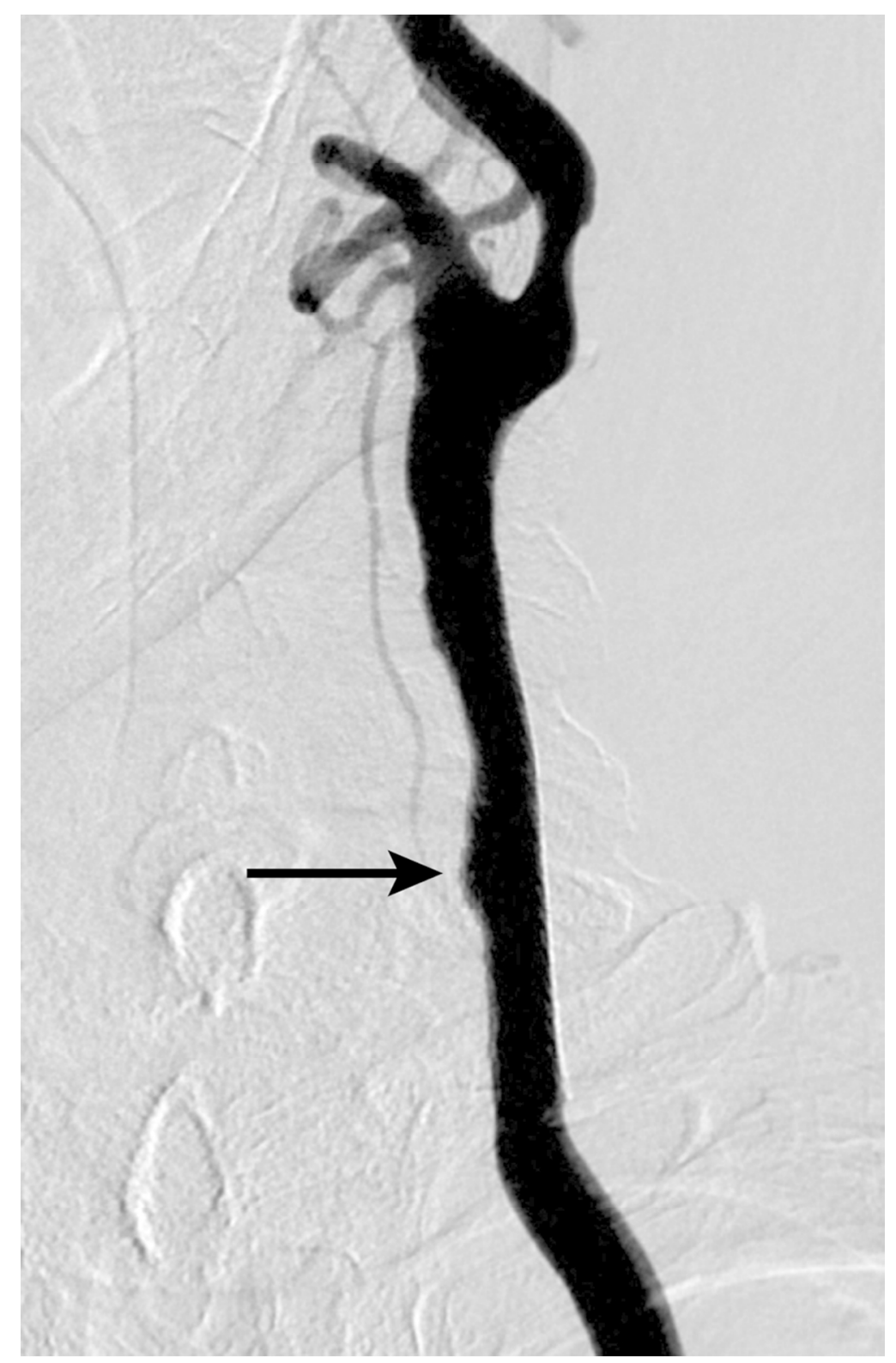

Figure 9D. 73 year old man with history of prior strokes, and who had also received radiation therapy for throat cancer. A, Sagittal spectral Doppler US demonstrates a high-grade stenosis within the left distal CCA with a peak systolic velocity of $469 \mathrm{~cm} / \mathrm{s}$. B, Severe CCA stenosis is confirmed on 3D reconstructed CTA (arrow). C, The stenosis is also demonstrated on DSA (arrow). The patient underwent successful CAS with angioplasty. D and $E$, Post-stent angiogram demonstrates a focal bulge in the location of the prior stenosis

(arrow), consistent with stent deformation without evidence of a fracture. A few days following the procedure, the patient had a transient ischemic attack. F, Grey scale US demonstrates the mildy deformed but intact stent struts. G, Sagittal color and spectral Doppler US of the proximal left ICA demonstrates interval appearance of a hypoechoic area at the origin of the left ICA (arrow) with an elevated peak systolic velocity of $191 \mathrm{~cm} / \mathrm{s}$ (scale not shown), consistent with an acute thrombus. H, Diffusion weighted MRI of the brain shows multiple hyperintense foci confined to the left cerebral hemisphere, consistent with multiple thromboemboli from a carotid source. 
$79 \times 123 \mathrm{~mm}(300 \times 300$ DPI $)$
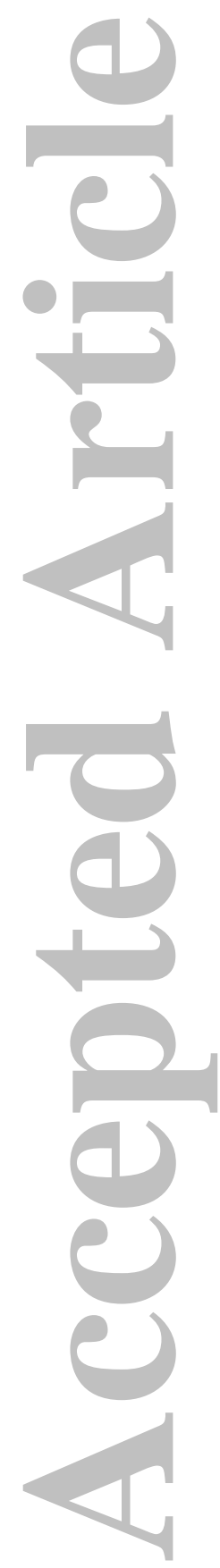

This article is protected by copyright. All rights reserved. 


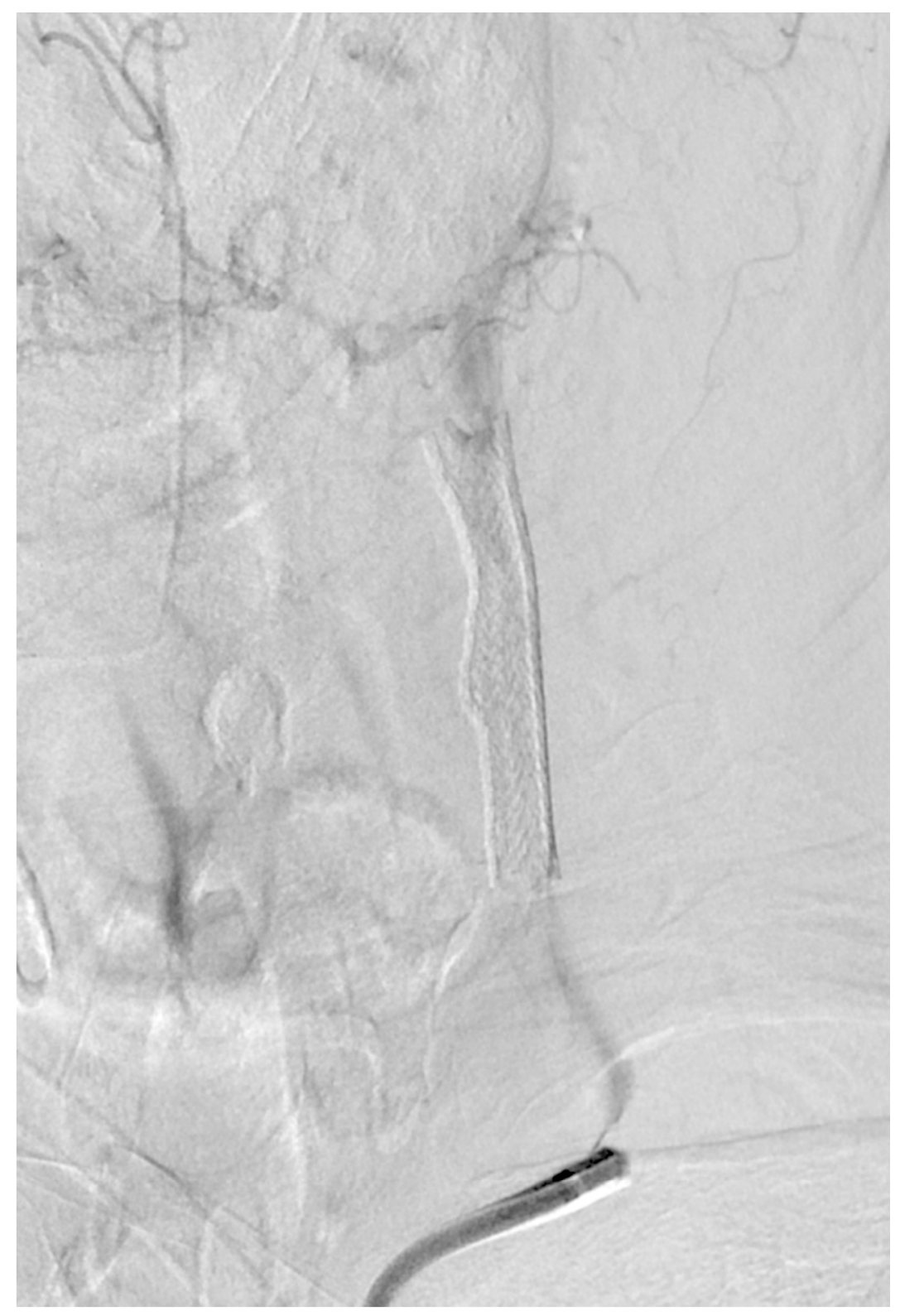

Figure 9E. 73 year old man with history of prior strokes, and who had also received radiation therapy for throat cancer. A, Sagittal spectral Doppler US demonstrates a high-grade stenosis within the left distal CCA with a peak systolic velocity of $469 \mathrm{~cm} / \mathrm{s}$. B, Severe CCA stenosis is confirmed on 3D reconstructed CTA (arrow). C, The stenosis is also demonstrated on DSA (arrow). The patient underwent successful CAS with angioplasty. D and $E$, Post-stent angiogram demonstrates a focal bulge in the location of the prior stenosis (arrow), consistent with stent deformation without evidence of a fracture. A few days following the procedure, the patient had a transient ischemic attack. F, Grey scale US demonstrates the mildy deformed but intact stent struts. G, Sagittal color and spectral Doppler US of the proximal left ICA demonstrates interval appearance of a hypoechoic area at the origin of the left ICA (arrow) with an elevated peak systolic velocity of $191 \mathrm{~cm} / \mathrm{s}$ (scale not shown), consistent with an acute thrombus. H, Diffusion weighted MRI of the brain shows multiple hyperintense foci confined to the left cerebral hemisphere, consistent with multiple thromboemboli from a carotid source. 
$79 \times 117 \mathrm{~mm}(300 \times 300$ DPI $)$
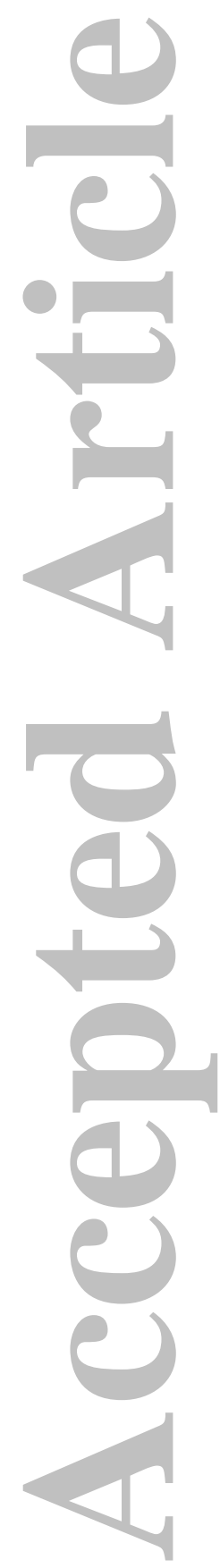

This article is protected by copyright. All rights reserved. 


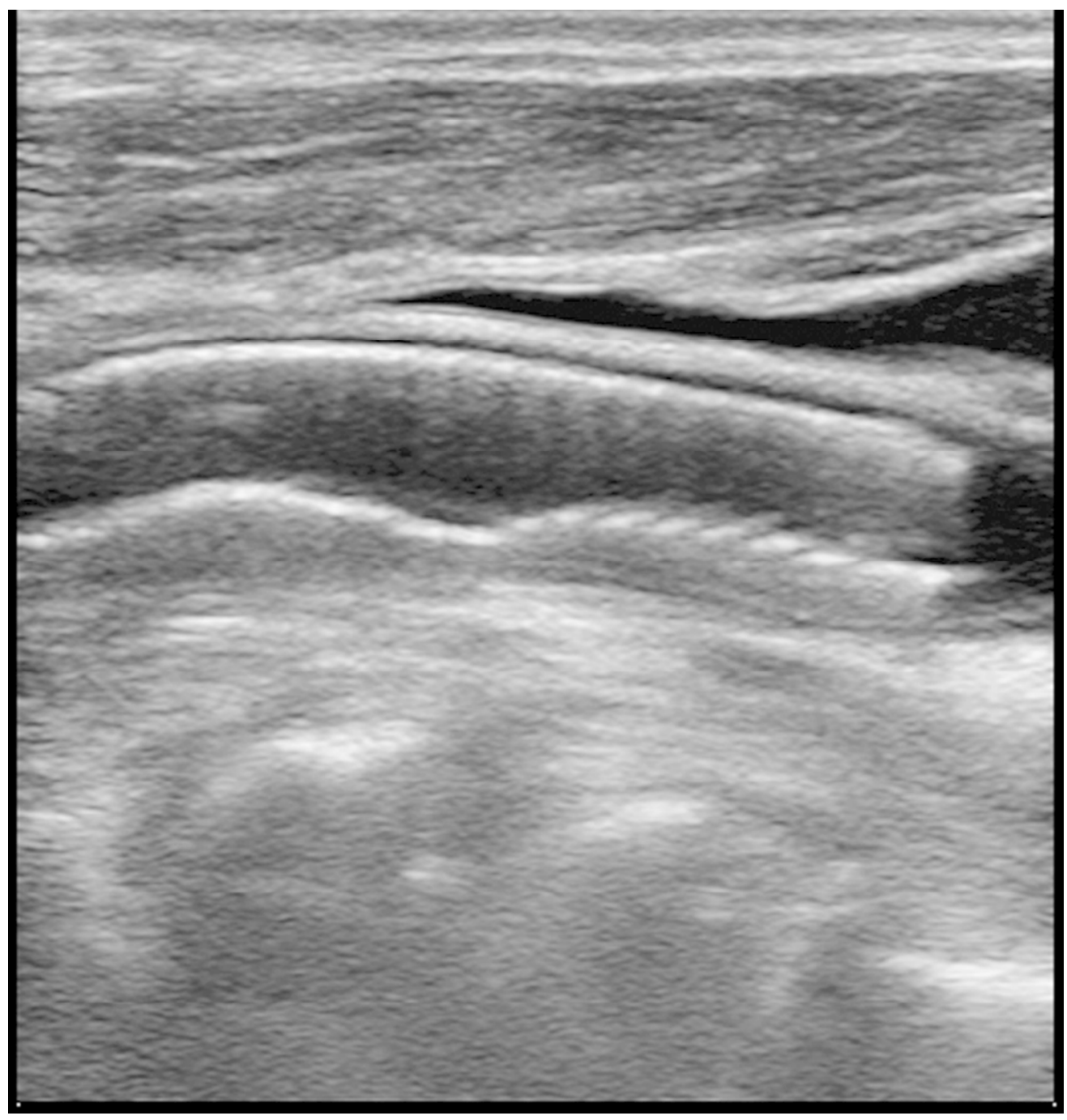

Figure 9F. 73 year old man with history of prior strokes, and who had also received radiation therapy for throat cancer. A, Sagittal spectral Doppler US demonstrates a high-grade stenosis within the left distal CCA with a peak systolic velocity of $469 \mathrm{~cm} / \mathrm{s}$. B, Severe CCA stenosis is confirmed on 3D reconstructed CTA (arrow). C, The stenosis is also demonstrated on DSA (arrow). The patient underwent successful CAS with angioplasty. D and $E$, Post-stent angiogram demonstrates a focal bulge in the location of the prior stenosis

(arrow), consistent with stent deformation without evidence of a fracture. A few days following the procedure, the patient had a transient ischemic attack. F, Grey scale US demonstrates the mildy deformed but intact stent struts. G, Sagittal color and spectral Doppler US of the proximal left ICA demonstrates interval appearance of a hypoechoic area at the origin of the left ICA (arrow) with an elevated peak systolic velocity of $191 \mathrm{~cm} / \mathrm{s}$ (scale not shown), consistent with an acute thrombus. H, Diffusion weighted MRI of the brain shows multiple hyperintense foci confined to the left cerebral hemisphere, consistent with multiple thromboemboli from a carotid source.

$79 \times 83 \mathrm{~mm}(300 \times 300$ DPI $)$ 


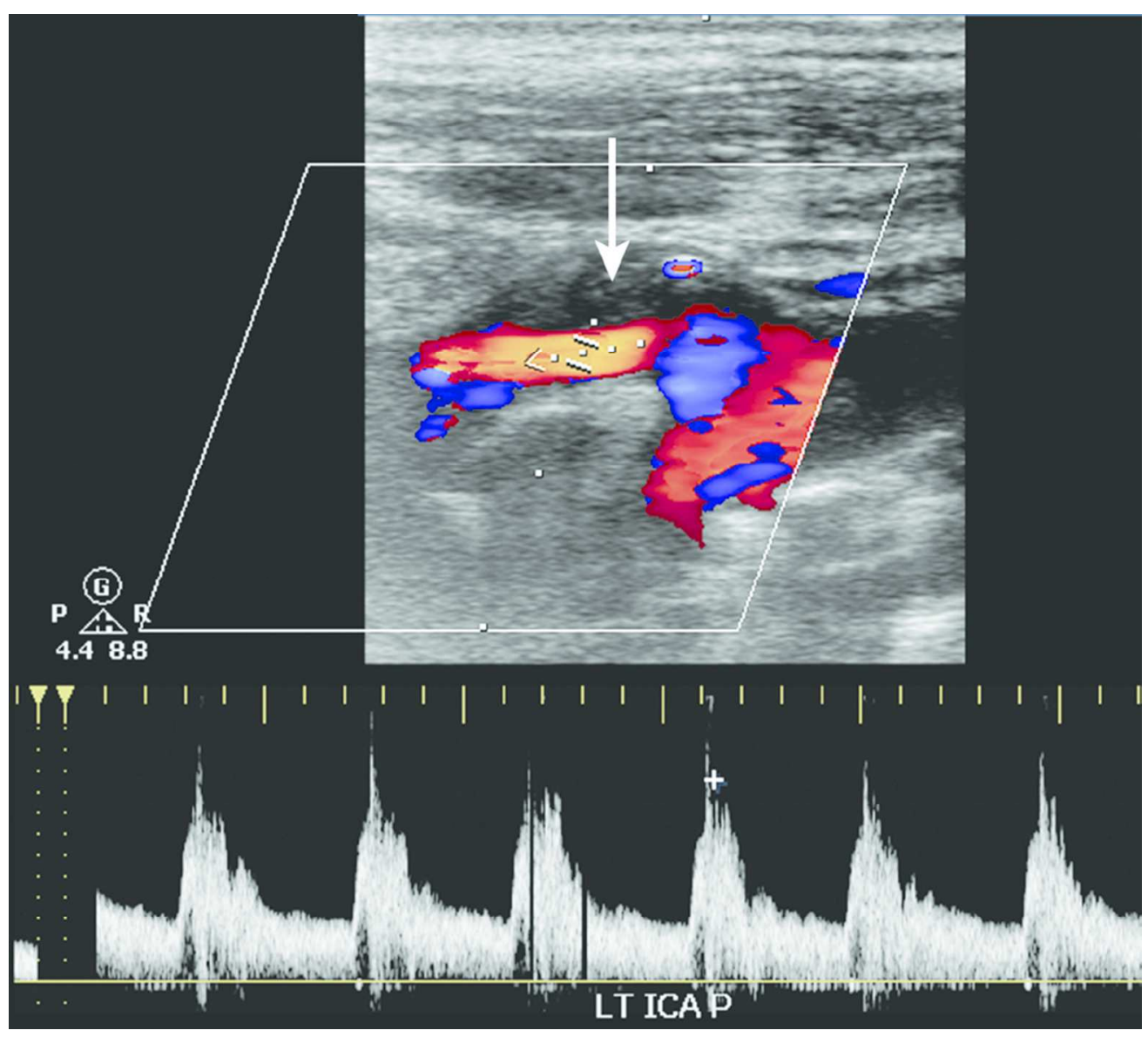

Figure 9G. 73 year old man with history of prior strokes, and who had also received radiation therapy for throat cancer. A, Sagittal spectral Doppler US demonstrates a high-grade stenosis within the left distal CCA with a peak systolic velocity of $469 \mathrm{~cm} / \mathrm{s}$. B, Severe CCA stenosis is confirmed on 3D reconstructed CTA (arrow). C, The stenosis is also demonstrated on DSA (arrow). The patient underwent successful CAS with angioplasty. D and $E$, Post-stent angiogram demonstrates a focal bulge in the location of the prior stenosis

(arrow), consistent with stent deformation without evidence of a fracture. A few days following the procedure, the patient had a transient ischemic attack. F, Grey scale US demonstrates the mildy deformed but intact stent struts. G, Sagittal color and spectral Doppler US of the proximal left ICA demonstrates interval appearance of a hypoechoic area at the origin of the left ICA (arrow) with an elevated peak systolic velocity of $191 \mathrm{~cm} / \mathrm{s}$ (scale not shown), consistent with an acute thrombus. H, Diffusion weighted MRI of the brain shows multiple hyperintense foci confined to the left cerebral hemisphere, consistent with multiple thromboemboli from a carotid source.

$79 \times 71 \mathrm{~mm}(300 \times 300$ DPI $)$ 


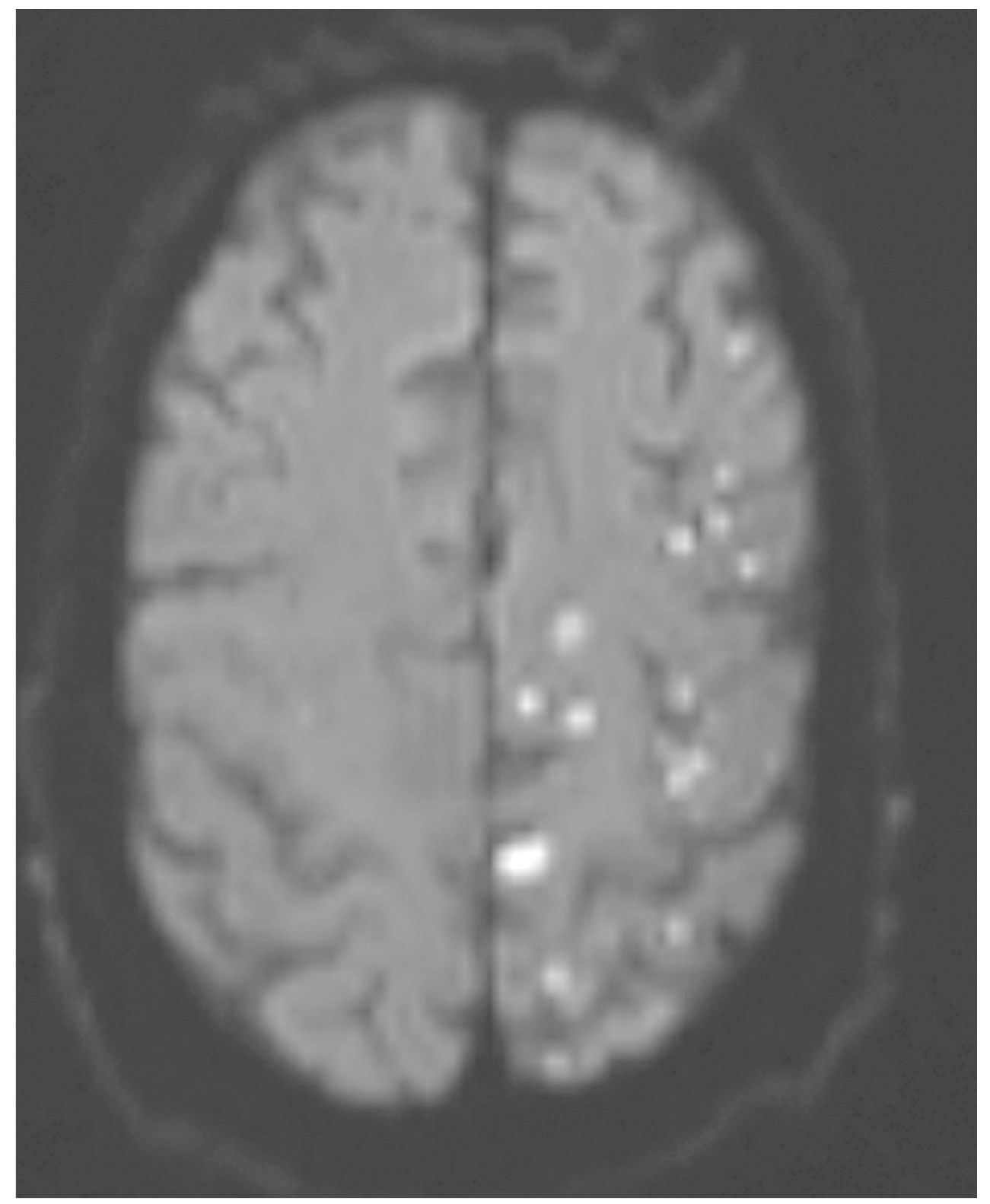

Figure 9H. 73 year old man with history of prior strokes, and who had also received radiation therapy for throat cancer. A, Sagittal spectral Doppler US demonstrates a high-grade stenosis within the left distal CCA with a peak systolic velocity of $469 \mathrm{~cm} / \mathrm{s}$. B, Severe CCA stenosis is confirmed on 3D reconstructed CTA (arrow). C, The stenosis is also demonstrated on DSA (arrow). The patient underwent successful CAS with angioplasty. D and $E$, Post-stent angiogram demonstrates a focal bulge in the location of the prior stenosis

(arrow), consistent with stent deformation without evidence of a fracture. A few days following the procedure, the patient had a transient ischemic attack. F, Grey scale US demonstrates the mildy deformed but intact stent struts. G, Sagittal color and spectral Doppler US of the proximal left ICA demonstrates interval appearance of a hypoechoic area at the origin of the left ICA (arrow) with an elevated peak systolic velocity of $191 \mathrm{~cm} / \mathrm{s}$ (scale not shown), consistent with an acute thrombus. H, Diffusion weighted MRI of the brain shows multiple hyperintense foci confined to the left cerebral hemisphere, consistent with multiple thromboemboli from a carotid source. 
$79 \times 98 \mathrm{~mm}(300 \times 300 \mathrm{DPI})$
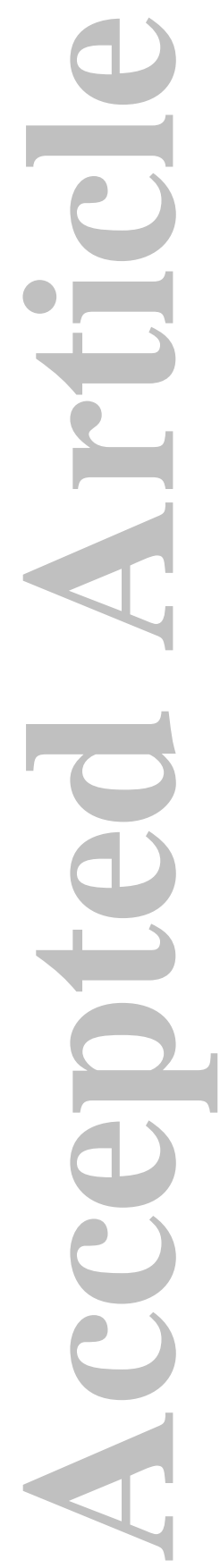

This article is protected by copyright. All rights reserved. 